\title{
Engineering of new hydrogel beads based conducting polymers: Metal-free catalysis for highly Organic Pollutants degradation
}

\author{
Abdellah AIT EL FAKIR ${ }^{1,2 *}$, Zakaria ANFAR ${ }^{1,2,3}$, Abdallah AMEDLOUS 4 , Mohamed ZBAIR, Zakaria \\ HAFIDI $I^{6}$, Mohammed EL ACHOURI ${ }^{6}$, Amane JADA ${ }^{2,3 *}$, Noureddine EL ALEM ${ }^{1}$
}

\begin{abstract}
1Laboratory of Materials \& Environment (L.ME). lbn Zohr University, Agadir, 80000, Morocco.
${ }^{2}$ Inctituta of Materials Srience of Multhousa (ISPM). Hauto Alsace Inniversity, Mulhouke, f810n, Franre.

${ }^{3}$ Strasbourg University, Strasbourg, 67081, France.

4Laboratory of Materials, Catalysis \&Valorization of Natural Resources (LMaCaVa). Hassan II University, Casablanca, 20650, Morocco.

${ }^{5}$ Laboratory of Catalysis \& Materials Corrosion (LCCM). Choualb Doukkali University, El Jadida, 24000, Morocco

${ }^{6}$ Laboratolre de physlco-chimie des matérlaux inorganiques et organiques, Centre des 16 Sciences des Matériaux, École Normale superieure-Rabat, Mohammed V University In Rabat, 17 Rabat, Morocco.
\end{abstract}

*Corresponding authors: aitelfakir.abdellah@gmail.com, amane.jada@uha.fr

\section{Abstract}

Engineering of new heterogeneous metal-free catalysts is of great importance for persulfate activation. Herein, Innovative conductive polymers Polyaniline (PANI) and Polypyrrole (PPy) were used as promising catalysts for PS activation to effectively degrade organic pollutants. In fact, PANI and PPY can provide the necessary electron transfer based on nitrogen atoms located in the conjugated chains compared to their counterparts in the defective sites. In addition, PANI and PPY were immobilized inside membrane formed by cross linking alginate biopolymer by divalent calcium ions, in order to develop a new stable hydrogel beads catalyst. The prepared hydrogels-activated PS process could maintain excellent catalytic properties, mitigate catalysts deactivation, increased chemical stability and offer hope for their application in the industrial scale. The radical scavenging experiments monitored by the electron paramagnetic resonance and functional theory calculations, indicated the role of the PANI and PPy conjugated chains and the nature of the reaction pathways.

Keywords: Conductive polymers; hydrogel beads; metal-free catalysts; persulfate (PS) activators. 


\section{Introduction}

The increasing concern about the environmental pollution and the run out of fossil fuel energies have urged researchers to seek renewable energy sources, new methods and functional materials for the pollutants removal [1-3]. Conventionally processes (e.g. adsorption, flocculation, membrane filtration and biological treatment, etc.) have been employed for the treatment of wastewater contaminated by organic pollutants. However, those already mentioned technologies suffer from some drawbacks mainly related to their low removal efficiency or to their non-destructive nature, generating hence secondary pollution (sludge formation). Advanced oxidation processes (AOPs) were demonstrated as powerful techniques to produce highly reactive oxygen species (ROS) from various superoxides for their uses in the toxic organic pollutants degradation [4,5]. Sulfate radicalbased advanced oxidation processes (SR-AOP) are becoming more and more popular due to their advantages, such as their redox potential (2.5-3.1 V vs NHE) which is higher than the hydroxyl radical $\left(\mathrm{OH}^{\circ}, 1.9-2.7 \mathrm{~V}\right.$ v $\left.\mathrm{NHE}\right)$, their high stability, their longer half-life $(30-40 \mu \mathrm{s})$ and their wide operating $\mathrm{pH}$ range (3-9). Most often $\mathrm{SO}_{4}{ }^{-}{ }^{-}$is generated by the activation of peroxymonosulfate (PMS) or persulfate (PS) $[6,7]$. Various activation methods were used for the PS activation including heat process, UV irradiation, transition metals, and ultrasound [8]. In particular, PS activation using transition metal-based heterogeneous catalysts (Co, Fe and $\mathrm{Mn}$ ) has received wide attention due to the resulted good catalytic performance for rapid decomposition of a wide range of contaminants and their recyclability [8]. However, these metal-based catalysts suffer from certain drawbacks such as their poor stability and their secondary pollution induced by the inevitable metal leaching during the catalytic process, limiting hence their use on an industrial scale. In recent years, metal-free catalysts, have taken attention of worldwide scientists due to their various applications from catalysis for energy production, as well as pollutants degradation, to energy storage. As an alternative, metal-free carbon based catalysts (carbocatalysts) such as graphene oxide [9], reduced graphene oxide [10], nanodiamond [11] and graphitic carbon nitride $\left(g-C_{3} N_{4}\right)$ [12], were widely studied as promising catalysts for PS and PMS activation. It is noteworthy to note that the unique properties of metal-free carbocatalysts (e.g. large surface area, high catalytic activity, superior biocompatibility and high stability, etc.), make these materials promising candidates for the AOPs applications. According to the experimental (Table S1) and the theoretical data reported 
in the literature, heteroatoms (e.g., N , S and P) could activate the inert carbon framework by increasing the charge density of adjacent carbon atoms, promoting hence the interaction occurring between the catalyst and the PS which results in the cleavage of the $\mathrm{O}-\mathrm{O}$ bond in the PS [7,13-15]. Importantly, nitrogen-doped carbon based materials have attracted broad interest, as catalysts which substantially alter the catalytic oxidation from a radical process to a non-radical pathway in PS and PMS activation towards efficient organic pollutants degradation $[6,16]$. In fact, nitrogen-doped carbon materials are synthesized by heat treatment of carbon-based materials in the presence of nitrogen precursors or by doping them during the synthesis procedure [17-19]. However, the synthesis of N-rich carbocatalysts is challenging due to the thermal instability of the desirable nitrogenous species (pyrrolic, pyridinic, and graphitic N). In addition, a smaller amount of nitrogen species in the carbocatalyst can lead to poor catalytic efficiency, preventing hence the practical applications of such catalysts $[16,18]$. Polyaniline (PANI) and polypyrrole (PPy) are nitrogen-containing conducting polymers and they are qualified candidates for several reasons. Firstly, PANI and PPy are typical electrically conductive polymers, and their conjugated chains can provide the necessary electron transfer. Secondly, as the nitrogen atoms are located in the conjugated chains, they are more stable than their counterparts in the carbocatalysts defective sites $[20,21]$. However, there are still has some drawbacks, such as catalyst recovery after reaction remains a major problem. To overcome these problems, the encapsulation of these conducting polymers in polysaccharide hydrogel beads is an effective approach to enhance their separation ability, to maintain their intrinsic properties and to preserve their long-term stability. Thus, as a continuation of our research efforts in designing and preparing sustainable catalysts for PS activation towards water decontamination, we report in the present work, the development of nitrogen-rich metal-free catalyst based on PANI and PPy encapsulated in alginate beads. The aim of the work is to synergistically combine the merit of each individual component in order to achieve the PS activation for the degradation of orange $G$ (OG) pollutant present in water. The characterizations of the synthesized materials were carried out by various methods such as Fourier Transform Infra-Red spectroscopy (FTIR), X-ray Photoelectron Spectroscopy (XPS), nitrogen adsorption/desorption, Scanning Electron Microscopy (SEM), Energy Dispersive Spectroscopy (SEM-EDS), Transmission Electron Microscopy (TEM) and Streaming Induced Potential (SIP). Further, the influence of various parameters including catalyst dosage, oxidant dosage and $\mathrm{pH}$ value, were investigated and 
discussed in detail. The mechanism of the PS activation was also investigated using probe reagents and Electron Paramagnetic Resonance (EPR).

\section{Experimental section}

\subsection{Chemicals reagents}

Aniline and pyrrole monomers were purchased from Sigma Aldrich and distilled before their uses. Ammonium persulfate (APS, $\left.\left(\mathrm{NH}_{4}\right)_{2} \mathrm{~S}_{2} \mathrm{O}_{8}\right)$, sodium hydroxide $(\mathrm{NaOH})$, hydrochloric acid $(\mathrm{HCl})(37 \%)$, sodium azide $\left(\mathrm{NaN}_{3}\right)$, tert-butanol (TBA), ethanol (EtOH), sodium persulfate (PS, $\mathrm{Na}_{2} \mathrm{~S}_{2} \mathrm{O}_{8}, 99 \%$ ), sodium alginate, orange $\mathrm{G}(\mathrm{OG})$, bisphenol A (BPA), sulfathiazole (STZ, 99\%), and rhodamine $\mathrm{B}(\mathrm{RhB})$, were provided by Sigma-Aldrich and were used as received. The $\mathrm{pH}$ values of the solutions were adjusted by using $\mathrm{HCl}$ and $\mathrm{NaOH}$ standard aqueous solutions (Merck Millipore).

\subsection{Preparation of polyaniline and Polypyrrole}

The polyaniline (PANI) was prepared following a simple polymerization method consisting in the use of common oxidation of the aniline monomer with a known ratio (aniline/APS). In short, $1 \mathrm{ml}$ of monomer aniline was dissolved in $30 \mathrm{ml}$ of $\mathrm{HCl}$ water solution $(0.1 \mathrm{M})$, then $20 \mathrm{ml}$ of $\mathrm{HCl}$ water solution $(0.1 \mathrm{M})$ containing the APS was added dropwise, and the resulting mixture was stirred, at room temperature, for 24 hours. Finally, the black product was collected by vacuum filtration, washed several times with distilled water and absolute ethanol, and then dried at $60^{\circ} \mathrm{C}$ in a vacuum oven for 6 hours.

The Polypyrrole (PPy) was prepared using easy polymerization process based on the oxidation of the pyrrole monomer with a known ratio (pyrrole/ $\mathrm{FeCl}_{3}, 6 \mathrm{H}_{2} \mathrm{O}$ ). Hence, $1 \mathrm{ml}$ of the pyrrole monomer was first dissolved in $30 \mathrm{ml}$ of distilled water solution, and then $20 \mathrm{ml}$ of distilled water solution containing $\mathrm{FeCl}_{3}, 6 \mathrm{H}_{2} \mathrm{O}$ was added dropwise to the pyrrole aqueous solution. Subsequently, the resulting mixed solution was stirred at room temperature for 24 hours. Finally, the product was collected by vacuum filtration, washed several times with distilled water and absolute ethanol. Finally, the obtained product was dried at $60{ }^{\circ} \mathrm{C}$ in a vacuum oven for 6 hours. 


\subsection{Synthesis of the core (PANI or PPy) - shell (alginate-calcium) beads: PANI@Alg and PPy@Alg}

Particles with the core (PANI or PPy)-Shell (alginate-calcium) used in this work were prepared according to the following protocol: $500 \mathrm{mg}$ of alginate powder was dissolved in 100 $\mathrm{ml}$ agitated for 4 hours, then $500 \mathrm{mg}$ of PANI or PPy was added to the mixture and left to stir for 3 hours. In addition, $\mathrm{CaCl}_{2}$ solution $(0.1 \mathrm{M})$ was placed in a crystallizer and kept under low agitation. The preparation of the hydrogel beads was assured by mixing both solutions of polymers/alginate and $\mathrm{CaCl}_{2}$, the preparation of beads was performed drop to taste using a pump. However, when each droplet of the alginate solution was placed in contact with the $\mathrm{Ca}^{2+}$ ions, a core (PANI/PPy)-shell beads (alginate and $\mathrm{Ca}^{2+}$ ) was then formed after incubation for several minutes (Fig. S1). As shown in Fig. S1, the beads were black and homogeneous with a uniform spherical shape, and the core-shell structure was clearly shown in the optical image. Finally, the beads were washed several times with distilled water and stored in a watery medium.

\subsection{Characterization}

The chemical structures of materials were studied by infrared spectroscopy using a Shimadzu 4800 S operating in the transmission mode. The FTIR spectra were recorded in the range of $400-4000 \mathrm{~cm}^{-1}$ at a resolution of $32 \mathrm{~cm}^{-1}$. All the spectra were obtained after an accumulation of 92 scans. Measurements of micro-Raman spectra were performed on a Raman spectroscopy system (DXR ${ }^{\mathrm{TM}} 2$, Thermo Fisher Scientific) in the range of $300-1800 \mathrm{~cm}^{-}$ ${ }^{1}$ with a $532 \mathrm{~nm}$ wavelength incident laser light. The X-ray diffraction spectra of the prepared materials were collected on a PANalytical MPD X'Pert Pro diffractometer operating with $\mathrm{Cu}$ $K \alpha$ radiation $(\lambda=0.15418 \mathrm{~nm}$ ). The $X$-ray Photoelectrons Spectroscopy (XPS) was carried out by using XPS VG SCIENTA, Model SES-200. The surface morphologies of the different materials were observed by using Scanning Electron Microscopy (SEM, FEI, model Quanta 400) and Transmission Electron Microscopy (TEM, Philips CM 200). The in situ electrostatic behavior of different systems were assessed by evaluating the solid surface electrical potential (Streaming Induced Potential, SIP) through the use of Müteck PCD 02 apparatus [22].

\subsection{Catalytic Activity Evaluation}

The catalytic activity of the prepared materials was evaluated by activating the PS for the $O G$ degradation. Thus, in a typical test, $50 \mathrm{~mL}$ of $O G$ aqueous solution (50 $\mathrm{mg} \mathrm{L}^{-1}$ ) was 
continuously agitated with $0.2 \mathrm{~g} / \mathrm{L}$ of catalyst for a contact time of $35 \mathrm{~min}$ to avoid confounding effects of adsorption. Then, the catalytic reaction was initiated by adding $4 \mathrm{mM}$ of PS into the mixture. During the test, aliquots were withdrawn from the reaction medium at given time intervals, then filtered through a $24 \mu \mathrm{m}$ syringe filter before their analyses. In order to identify the reactive oxygen species generated in the catalytic reaction, amounts of radical scavengers such as ethanol (EtOH) and tert-butyl-alcohol (TBA), were added in the reaction medium to trap $\mathrm{SO}_{4}{ }^{\circ-}$ and $\mathrm{HO}{ }^{*}$, respectively. In addition, sodium azide $\left(\mathrm{NaN}_{3}\right)$ was used as quencher for ${ }^{1} \mathrm{O}_{2}$. It should be noted that the experimental tests were performed in duplicate or triplicate, the mean values of the measured parameters were determined, and their error bars were assessed, and they were represented in the corresponding graphs.

\subsection{Analytical Methods}

The OG concentration was analyzed by UV-Vis spectrophotometer (6705 UV/Vis JENWAY) at wavelength of $\lambda=478 \mathrm{~nm}$. In order to study the mineralization of the pollutants, the total organic carbon (TOC) was measured using TOC-L SHIMADZU analyser. Electron Paramagnetic Resonance (EPR) measurement was carried out on a Bruker EMX-plus X-band spectrometer to detect the reactive oxygen species generated during the PS activation. The $\mathrm{N}$-tert-butyl- $\alpha$ phenylnitrone (PBN) was used as a spin-trapping agent for $\mathrm{SO}_{4}{ }^{\circ-}$ and $\mathrm{HO}^{\circ}$ and 2,2,6,6tetramethyl-4-piperidone (TEMP) as a spin-trapping agent for ${ }^{1} \mathrm{O}_{2}$. The points of zero charges (PZC) of the PANI and the PPy, were determined by the salt addition method according to the method described by Tahira et al., 2011 [23]. Suspensions of $0.2 \mathrm{~g}$ of PANi and PPy catalysts were put into contact with $40 \mathrm{~mL}$ of $\mathrm{NaNO}_{3}(0.1 \mathrm{M})$. Then, the $\mathrm{pH}$ of the suspension was adjusted to an initial pH value ranging between 2 and 10, by using $\mathrm{HNO}_{3}(0.1 \mathrm{M})$ and $\mathrm{NaOH}$ $(0.1 \mathrm{M})$ aqueous solutions. The mixtures were stirred for $24 \mathrm{~h}$ (to ensure homogenization of the suspension) at $250 \mathrm{rpm}$ until the $\mathrm{pH}$ was kept constant. All the experiments were performed in a thermostated vessel at $\left(20 \pm 0.2^{\circ} \mathrm{C}\right)$.

The Computational methodology (DFT) has been shown in the Text S1 Supplementary Material. 


\section{Results and discussion}

\subsection{Physicochemical Structures}

The XPS analysis was performed to study the elemental composition and the chemical state of the as-prepared PANI and PPy. The XPS survey spectra of PANI and PPy are displayed in Fig. 1a. As can be seen in this figure, the XPS survey of PANI shows the presence of carbon (C1s), oxygen (O1s, OKLL), nitrogen (N1s) as well as sulfur (S2p, S2s). The XPS survey of PPy shows the presence of the characteristic peaks of carbon (C1s), oxygen (O1s, OKLL), nitrogen ( $\mathrm{N}(1 \mathrm{~s})$ and $\mathrm{Cl} 2 \mathrm{p}$ Fig. 1d. The presence of chlorine in the surface of PPy indicates the positively charged PPy, which was balanced by the $\mathrm{Cl}^{-}$anion. In addition, the presence of oxygen atoms in PANI and PPy should probably result from certain adsorbed impurities on the polymer surface (e.g., adsorbed $\mathrm{CO}_{2}$ and small molecules). The $\mathrm{C} 1 \mathrm{~s}$ spectrum of PANI (Fig. 1b) can be deconvoluted in four peaks centred at, 288.02, 286.49, 285.15 and $284.32 \mathrm{eV}$, which are attributed to $\mathrm{C}=\mathrm{O}, \mathrm{C}-\mathrm{O}, \mathrm{C}-\mathrm{N} / \mathrm{C}=\mathrm{N}$ and $\mathrm{C}-\mathrm{C} / \mathrm{C}-\mathrm{H}$ bonds, respectively [24]. The N1s spectrum of PANI (Fig. 1C) was fitted into four component peaks centred at 401.99, 400.72, 399.58 and $398.34 \mathrm{eV}$, which are due to the presence of species such as imine $-\mathrm{NH}_{2}{ }^{+}-$, amine with a positive charge $=\mathrm{NH}^{+}-$, benzoic amine $-\mathrm{NH}-$ and quinoid imine $=\mathrm{N}-$, respectively [25] Regarding the XPS analysis of PPy, The C 1s spectrum of PPy (Fig. 1d) can be deconvoluted into three peaks at binding energy of $287.86,285.45$ and $284.95 \mathrm{eV}$, corresponding to, the $\mathrm{C}-\mathrm{O}$, $\mathrm{C}-\mathrm{N}$ and $\mathrm{C}-\mathrm{C}$ bonds, respectively [26]. The deconvolution of $\mathrm{N} 1 \mathrm{~s}$ spectrum of PPy (Fig.1e) shows three peaks centred at binding energy of $400.78,400.03$ and $398.25 \mathrm{eV}$, corresponding to nitrogen bond $=\mathrm{N}-$, nitrogen neutral pyrrole bond $(-\mathrm{N}-\mathrm{H}-)$, and positively charged nitrogen bonds $=\mathrm{N}^{\delta+} / \mathrm{C}-\mathrm{N}^{\delta+}$, respectively [26]. 

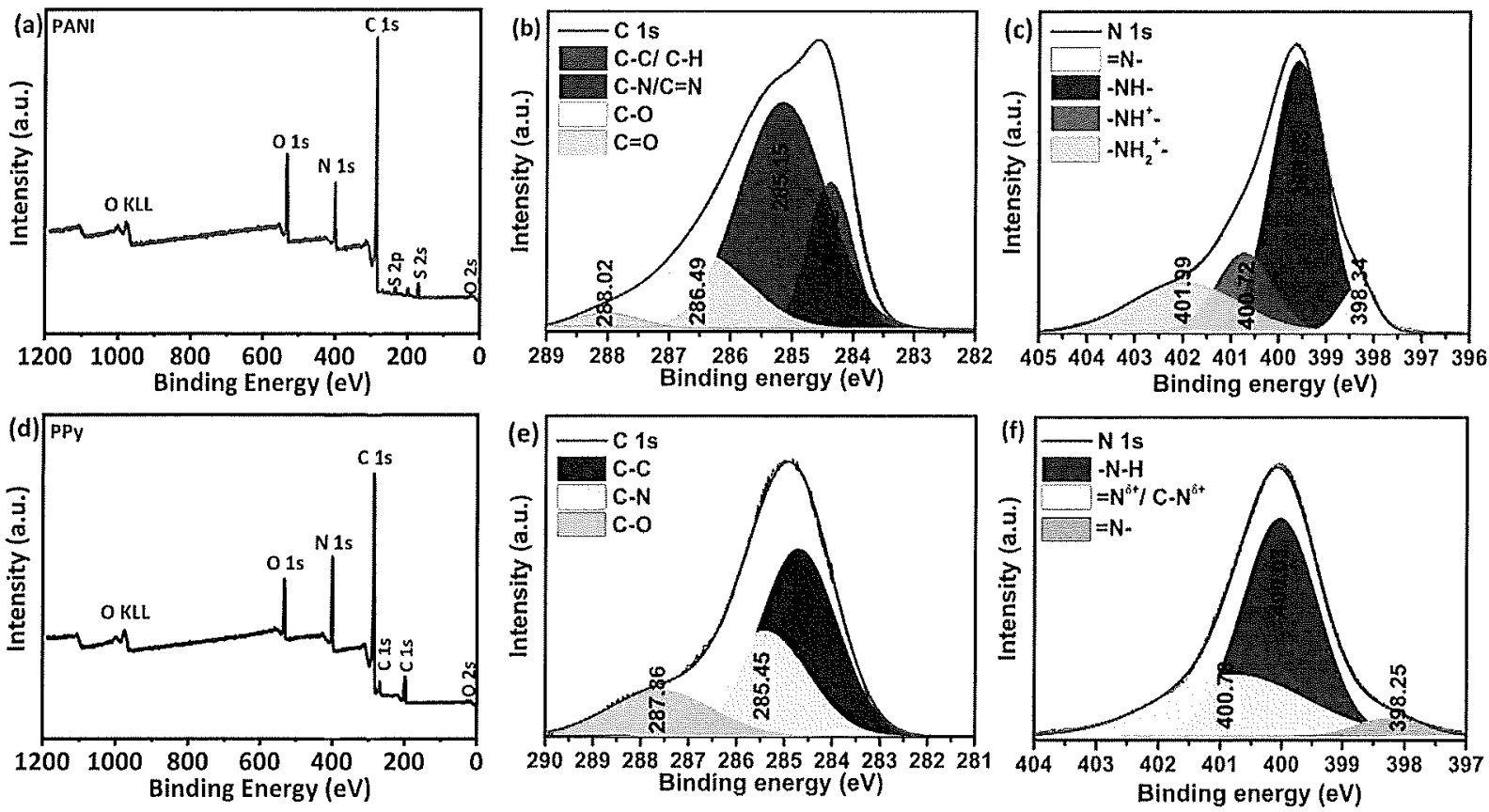

Fig. 1. (a,d) XPS survey spectrum of the PANI and PPy, High-resolution XPS spectra of (b) C1s of PANI; (c) N1s of PANI; (e) C1s of PPy and (f) N1s of PPY

The morphologies of PANI and PPy materials were studied by SEM and TEM analyses as displayed in Fig. 2a-d. The SEM images of PANI (Fig. 2a,a') reveal the presence of irregular and spherical nanometric particles. On the other hand, the SEM micrographs of PPy (Fig. 2b, $\mathbf{b}^{\prime}$ ) show spherical shape and narrow size distribution consisting of nanoparticles with a mean size of about $50 \mathrm{~nm}$. The TEM images of PANI (Fig. 2c, c') show completely featureless, highly disordered and completely amorphous material. These findings are in very good agreement with the powder X-ray diffraction data. In the case of PPy, the TEM analysis shows agglomerate spherical particles (Fig. 2d,d'). To investigate deeply the structural, as well as the chemical functionalities of the prepared catalysts (PANI and PPY), we carried out FTIR analyses, as shown in (Fig.2e). For the FTIR PPy spectrum, the characteristic bands centered at 1550 and $1461 \mathrm{~cm}^{-1}$, were attributed to the $\mathrm{C}=\mathrm{C}$ stretching and $\mathrm{C}-\mathrm{N}$ vibrations in the PPy ring [27]. The bands at 1310 and $1175 \mathrm{~cm}^{-1}$ are attributed to the $\mathrm{C}-\mathrm{H}$ bond, in the vibration plane and out of the vibration plane of the PPy ring, respectively. In addition, the band centered at $905 \mathrm{~cm}^{-1}$ is attributed to $\mathrm{C}-\mathrm{N}$ asymmetric stretching. The bands located at 1041 and $785 \mathrm{~cm}^{-1}$ are attributed to $\mathrm{N}-\mathrm{H}$ wagging [26]. The FTIR PANI spectrum shows many bands including 1550 and $1496 \mathrm{~cm}^{-1}$, which are attributed to $\mathrm{C}=\mathrm{C}$ stretching vibrations of quinoid and benzenoid rings, respectively. The bands at 1301 and $1150 \mathrm{~cm}^{-1}$ are due to the $\mathrm{C}-\mathrm{N}$ stretching of 
secondary aromatic amine, whereas the characteristic band at $1123 \mathrm{~cm}^{-1}$ is due to $\mathrm{C}-\mathrm{H}$ in plane bending of the aromatic ring, and out-of-plane $\mathrm{C}-\mathrm{H}$ deformation of disubstituted benzene ring appearing in $805 \mathrm{~cm}^{-1}$. The overall FTIR data give further evidence for the success of the polymerization reaction of the aniline [28]. To get further information on the catalyst structures, the Raman spectra of PANI and PPy were measured and they are depicted in Fig.2e. As can be observed in figure, the PPy spectrum shows certain peaks centred at 1578 and 1323 $\mathrm{cm}^{-1}$ which are due to, $\mathrm{C}=\mathrm{C}$ backbone stretching and antisymmetric $\mathrm{C}-\mathrm{N}$ stretching, respectively, whereas the peak at $1053 \mathrm{~cm}^{-1}$ is attributed to the deformation in the $\mathrm{C}-\mathrm{H}$ plane (polarons). The vibration mode at $971 \mathrm{~cm}^{-1}$ corresponds to the out-of-plane $\mathrm{C}-\mathrm{H}$ deformation of the quinoid form. In addition, the Raman PANI spectrum (Fig. 2f) shows main characteristic peaks, centred at $1167 \mathrm{~cm}^{-1}$ ( $\mathrm{C}-\mathrm{H}$ bending of the quinoid ring), at $1257 \mathrm{~cm}^{-1}$ (C-H bending of the benzene ring), at $1339 \mathrm{~cm}^{-1}$ (C-N stretch), and both bonds at $1501 \mathrm{~cm}^{-1}$ and $1597 \mathrm{~cm}^{-1}$ due to $C-C$ stretch of the benzene ring [29]. The $N_{2}$ adsorption-desorption isotherms of the starting samples PANI and PPy are shown in (Fig. S3a), and they were measured at $-196{ }^{\circ} \mathrm{C}$, after degassing the samples at $80^{\circ} \mathrm{C}$ during 24 hours. The calculated BET surface areas of PPY and PANI polymers are, respectively, $50 \mathrm{~m}^{2} / \mathrm{g}$ and $15 \mathrm{~m}^{2} / \mathrm{g}$. As can be observed in Fig. S3a, open hysteresis loops were observed for all samples and it is more pronounced for the PPy sample. Such open hysteresis loop appears to be typical to the slit pores morphology, while the micropores are cylindrical, confirming hence the fact that the opening of the hysteresis loops seems to be related to the "slit" shape of the mesopores (Table S2). Fig. S3b show the XRD spectra of the PANI and PPy catalysts. The diffractogram of PANI shows two peaks centred at $2 \theta=20.15^{\circ}$ and $2 \theta=25.75^{\circ}$, corresponding to the periodicities parallel and perpendicular to the polymer chain, respectively. This result indicates the amorphous nature of the PANI sample [29]. On the other hand, the XRD pattern of PPy shows a broad peak centred at $2 \theta=25^{\circ}$ which is due to the (002) Bragg reflection of the PPy chains [30]. 

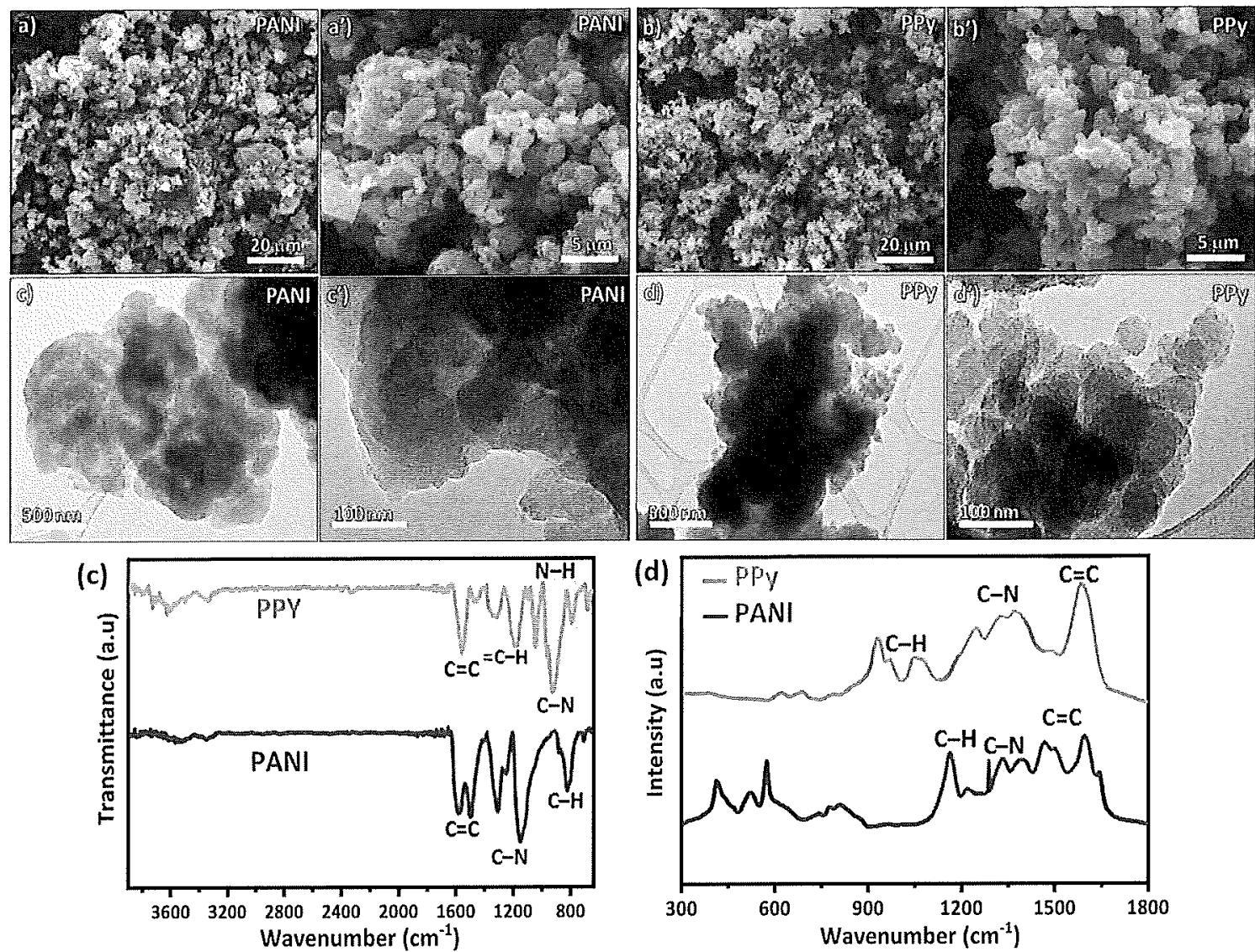

Fig.2. SEM and TEM images of PAN (a, $a^{\prime}$ and $\left.c, c^{\prime}\right)$ and PPy $\left(b, b^{\prime}\right.$ and $\left.d, d^{\prime}\right)$; FTIR spectra (c), Raman spectra (d) of PANI and PPY;

\subsection{Characterization and Mechanism of the core (PANI or PPy) - shell (alginate- calcium) beads formation: PANI@Alg and PPy@Alg}

\subsubsection{Characterization of hydrogel beads}

The preparation of PANI or PPy/Alginate beads (PANI@Alg and PPy@Alg) were confirmed by FTIR spectroscopy and SEM analyses. Thus, as can be seen in Fig.3a, the FTIR spectrum of alginate beads displayed peaks around 1037, 1515, 1625 and $3330 \mathrm{~cm}^{-1}$ which are attributed to - $\mathrm{C}-\mathrm{O}-\mathrm{C}$ - binding and -COO- (symmetric), -COO- (asymmetric) and stretching of $\mathrm{O}-\mathrm{H}$, respectively [31]. After encapsulation of PANI in alginate matrix, new peaks appeared corresponding to the FTIR PANI spectrum and a new peak appeared in the region of $1715 \mathrm{~cm}^{-}$ ${ }^{1}$ due to the asymmetric stretching of the -COO- alginate groups. This peak, which specifies the ionic bond, shows a large shift towards higher values with a decrease in intensity. In addition, the protonated $-\mathrm{NH}_{2}{ }^{+}$- amine groups of PANI replace the alginate sodium ions, modifying hence the charge density, the radius and the atomic weight of the cation [32]. On the other hand, the PPy@Alg spectrum (Fig. 3b) shows the respective characteristic peaks of 
the PPy polymer and the alginate matrix. In addition, the -COO- peak of alginate was shifted, as resulting from the electrostatic interaction of these groups with the polarized amine $-\mathrm{NH}^{+}$ belonging to PPy. The overall FTIR results confirm that the PANI and PPy conducting polymers were successfully introduced onto the Alginate surface [33].The SEM analysis coupled with the EDS mapping was performed to study the morphologies and the chemical composition of the PANI@Alg and the PPy@Alg beads. Hence, the SEM images of the beads (Fig. 3c-d) show that the PANI and PPy polymers were immobilized inside a layer formed by alginate and calcium ions, allowing the construction of the spherical beads. In addition, the Mapping images confirm these results. As can be observed in Fig.3c-d, the calcium ions are concentrated on the surface of the beads. In this way and in order to highlight the bead structure. the electrostatic interactions occurring at the particle surface were assessed by determining the Streaming Induced Potential (SIP).
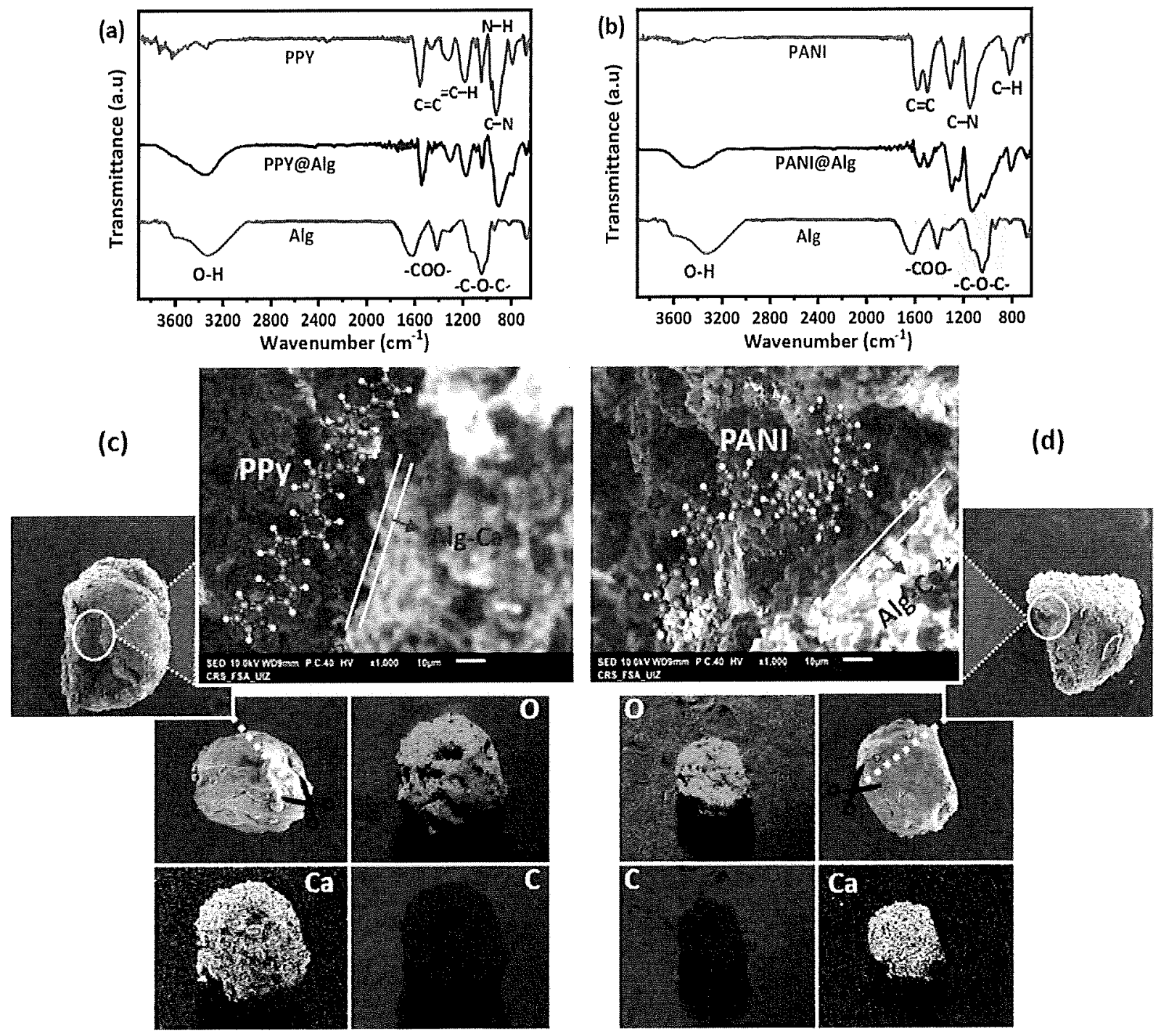
Fig. 3. FTIR spectra of Alginate, PPY and PPy@Alg (a) Alginate, PANI and PANI@Alg (b); SEM images and Mapping of the prepared beads PPy@Alg (c) and PANI@Alg (d)

\subsubsection{Investigation of the various Interactions occurring in the hydrogel beads}

The electrostatic interactions occurring between various systems, such as PANI, PPy, alginate and $\mathrm{Ca}^{2+}$ were studied. The SIP results show high electrostatic interactions between PANI/alginate and PPy/Alginate systems. Therefore, the chemical process of formation of these beads has been detailed using the following successive titrations:

- Titration 1 (Fig. 4a): Titration of an alginate solution $\left(5 \mathrm{~g} \mathrm{~L}^{-1}\right)$ with $\mathrm{Ca}^{2+}$ ions $(0.1 \mathrm{M})$. The titration shows a strong electrostatic interaction occurring between the carboxylic groups of the alginate and the $\mathrm{Ca}^{2+}$ ions. In the absence of $\mathrm{Ca}^{2+}$ ions, a SIP value is about $-442 \mathrm{mV}$ for the alginate. Afterward the addition of $\mathrm{Ca}^{2+}$ ions, the SIP value decreased and reached a plateau value in which the SIP $=0 \mathrm{mV}$.

- Titration 2 (Fig. 4a): Titration of an alginate solution ( $\left.5 \mathrm{~g} \mathrm{~L}^{-1}\right)$ by PANI and PPy $\left(5 \mathrm{~g} \mathrm{~L}^{-1}\right)$. Fig. $4 a$ indicates high electrostatic interactions between alginate/ $\mathrm{Ca}^{2+}$ in comparison to the alginate/PANI or to the alginate/PPy systems. The normalized SIP values, reached at the plateaux for alginate $/ \mathrm{Ca}^{2+}$, alginate/PANI, and alginate $/ \mathrm{PPy}$ systems, are $\mathrm{SIP}=-0.1, \mathrm{SIP}=-0.39$, and $\mathrm{SIP}=-0.3$, respectively.

- Titration 3 (Fig. 4b): Titration of a mixed solution of either PANI@Alg (ratio 1/1) or PPy@Alg (ratio 1/1), with $\mathrm{CaCl}_{2}(0.1 \mathrm{M})$. For this purpose, the titration of PANI@Alg and PPy@Alg systems by $\mathrm{CaCl}_{2}$, was started at the end of the alginate/PANI and the Alginate/PPy titrations. The results show important electrostatic interactions leading to low normalized SIP values and indicating stronger interactions occurring in the ternary systems (Polymer@Alginate/ $\mathrm{Ca}^{2+}$ ), in comparison to the binary systems (Alginate/ $\mathrm{Ca}^{2+}$ or Alginate/Polymer). Note that the electrostatic interaction occurring at alginate/polymer interface results mainly from the interaction between the hydrogen bound to nitrogen in the polymer chain and the alginate carboxylate groups, as depicted in Fig. 4c. On the other hand, complexation reaction occurs between alginate carboxylate groups and the calcium divalent cations. Furthermore, the difference in the behaviours observed in Fig. 4a between PANI and PPy towards the alginate surface charge screening, results likely from the difference in the polymer specific surface area and/or the polymer PZC value. Thus, both PANI and PPy are less negatively charged than 
alginate, however, PPy having higher surface area and PZC values as compared to PANI, seems to be more surface charged.

From the above SIP results, it can be concluded that there are important electrostatic interactions occurring between alginate, calcium ions $\left(\mathrm{Ca}^{2+}\right)$, PANI and PPy, leading hence, to the formation of the core-shell structure. Therefore, the core made of PANI or PPy, and coated with alginate shell cross-linked by calcium ions (Fig.4c).

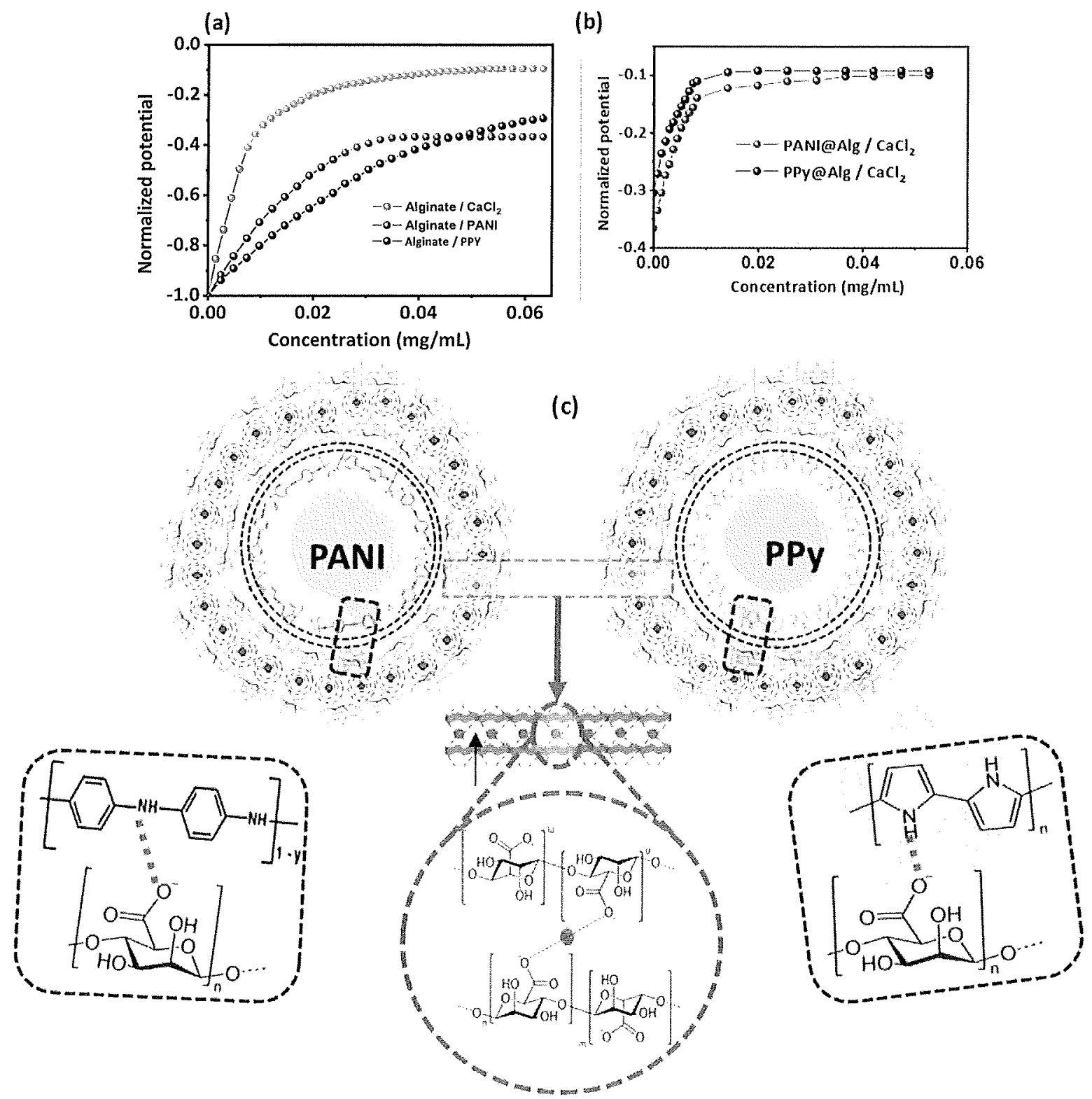

Fig. 4. Titration of the alginate $\left(5 \mathrm{~g} \mathrm{~L}^{-1}\right)$ by aqueous solutions of $\mathrm{CaCl}_{2}(0.1 \mathrm{M})$, PANI $\left(5 \mathrm{~g} \mathrm{~L}^{-1}\right)$ and PPy $\left(5 \mathrm{~g} \mathrm{~L}^{-1}\right)(\mathrm{a})$; Titration of the PANI@Alg mixture and PPy@Alg mixture by aqueous solution of $\mathrm{CaCl}_{2}$ $(0.1 \mathrm{M})$ (b); Chemical mechanism of the beads formation for PANI@Alg and PPy@Alg systems(c). 


\subsection{Catalytic OG degradation}

The catalytic activities of the prepared materials were evaluated for the PS activation towards the OG degradation. As displayed in Fig. 5a, when the PANI and PPy were used alone, the removal of OG is less than $20 \%$ and $16 \%$, respectively, confirming hence the negligible physical adsorption of the OG molecules on the polymer surface. For a heterogeneous catalytic reaction, the adsorption of OG on the surface of PANI and PPy is a preliminary step. The adsorption kinetic was studied in order to elucidate the adsorption mechanism. After 35 min of adsorption reaction, the stabilization plateau is reached indicating that the active sites are saturated (Fig.S4a). The value of adsorption capacity was calculated to be 61.5 and $45 \mathrm{mg}$

$\mathrm{g}^{-1}$ for PANI and PPy, respectively. In addition, when PS was used alone (without catalyst), only $5 \%$ of OG removal was achieved in $30 \mathrm{~min}$ by direct oxidation, indicating that the OG dye was difficult to oxidize by PS alone without using catalyst. However, when PS and the prepared catalysts (PANI or PPy) were combined, the degradation efficiency of OG was 100\%, in 30 min for PANI, and 35 min for PPy. These results indicate that PANI and PPy have excellent catalytic activities for the PS activation to produce active species with a strong oxidative capacity for the decomposition of the aromatic organic pollutants (such as OG) into small molecules until their final mineralization to carbon dioxide $\left(\mathrm{CO}_{2}\right)$ and water $\left(\mathrm{H}_{2} \mathrm{O}\right)$. It should be emphasized that the structures of the conductive polymers used in this study are rich in nitrogen and have stable $\mathrm{N}$ sites, which can provide a good and durable active site towards the PS activation. In addition, the higher electronegativity of nitrogen atoms $(\chi N=3.04)$ compared to carbon atoms $(\chi C=2.55)$ gives greater electron transfer capacity, and increases the charge density of adjacent carbon atoms, which promote interaction between catalysts active sites rich in electrons and persulfate molecules to produce reactive oxygen species [34].The catalytic activity of PANI and PPY were also compared with some common heterogeneous metal-based catalysts including $\mathrm{Fe}_{3} \mathrm{O}_{4}, \mathrm{CO}_{3} \mathrm{O}_{4}$. The results show low efficiencies of $\mathrm{Fe}_{3} \mathrm{O}_{4}$ and $\mathrm{CO}_{3} \mathrm{O}_{4}$ catalysts compared to PANI and PPy. Moreover, these materials have obvious metal ions leaching in solution, which is considered as a secondary pollution that can influence water quality [3537]. Furthermore, the catalytic activities of PANI and PPy were also evaluated for the PS activation towards removal of others organic contaminants such as RhB, STZ and BPA. As shown in Fig. 5b, the degradation rate of most pollutants can surpass $80 \%$ within 60 min. Accordingly, both PANI and PPy catalysts investigated in the present study can promote the 
decomposition of the PS to generate active species for the oxidation of wide range of organic pollutants. In order to evaluate the catalytic activities of catalysts in realistic conditions, both PANI and PPy were studied for PS activation towards OG degradation in river water (RW). As shown in Fig.5c, the degradation rates of OG in RW were 81 and 78\% for PANI and PPy, respectively. The negative impact for OG removal in RW medium could be attributed the high levels of chloride ions $\left(\mathrm{Cl}^{-}\right)$and the presence of organic matter, which would limit the catalytic performance. The total organic carbon (TOC) is important parameter in the evaluation of the organic pollutants degradation, because the spectroscopic methods cannot detect intermediates formed after degradation. As shown in Fig. S4b, the TOC removal reached values of 74, 78, 47 and 53\%, for PANI,PPy, PANI@Alg and PPy@Alg, respectively. The obtained results suggest that these materials have excellent catalytic performance to efficiently transform and mineralize $\mathrm{OG}$ into $\mathrm{CO}_{2}$ and $\mathrm{H}_{2} \mathrm{O}$.

After encapsulation of both PANI and PPy with alginate to form PANI@Alg and PPy@Alg beads. The degradation efficiency of OG can reach 95\% within 120 min for PPy@Alg, and about 150 min for PANI@Alg (Fig. 5d). In this case, the diffusion process of OG molecules inside the membranes of beads becomes difficult, and the presence of negatives functional groups on alginates $(-\mathrm{COOH}$ and $\mathrm{OH})$ act as donors towards some positive active nitrogen sites of PANI or PPy. Therefore, the time of removal of OG increased from $35 \mathrm{~min}$ to $120-150 \mathrm{~min}$. However, encapsulation of prepared catalysts by this biopolymer can improve their chemical stability as well as their separation ability. 
(a)

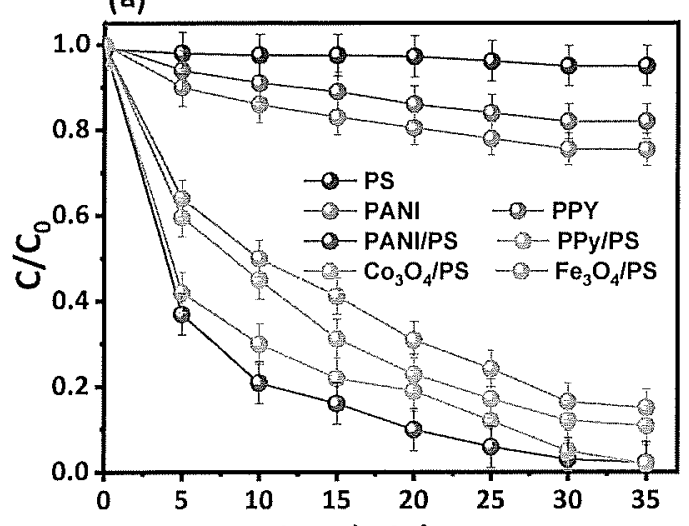

(c)

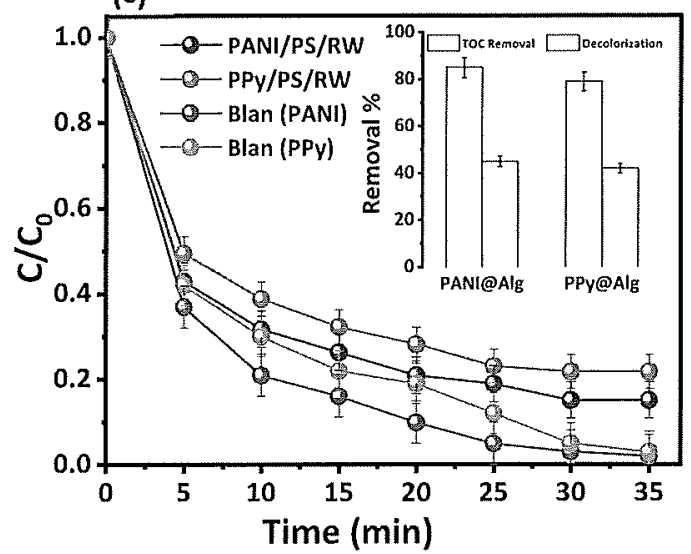

(b)

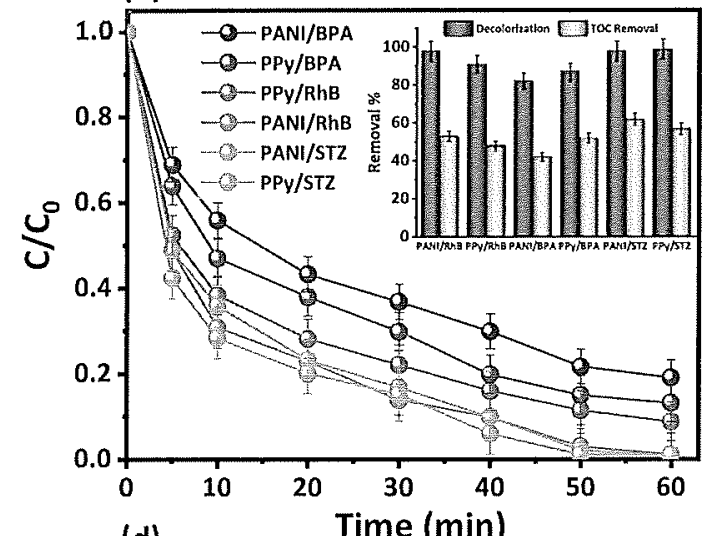

(d)

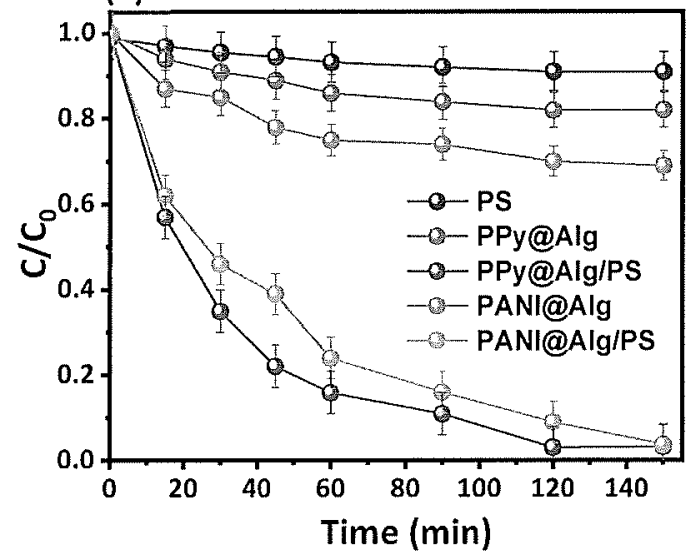

Fig. 5. Degradation of OG: with PANI and PPy(a); Degradation of BPA and RhB: with PANI and PPy (b). Effect of water matrix (c), Degradation of OG with PANI@Alg and PPy@Alg (d) ([Catalyst] $=0.2 \mathrm{~g} \mathrm{~L}^{-1},[\mathrm{OG}]=50 \mathrm{mg} \mathrm{L}^{-1},[\mathrm{PS}]=4 \mathrm{mM} ;[\mathrm{BPA}]=10 \mathrm{mg} \mathrm{L}^{-1} ;[\mathrm{RhB}]=20 \mathrm{mg} \mathrm{L}^{-1}$; [STZ] $=20 \mathrm{mg} \mathrm{L}^{-1}$ )

\subsection{Effect of Experimental Parameters}

Fig. 6 shows the important factors affecting the degradation efficiency of OG including catalyst dose, PS concentration, $O G$ concentration, initial $\mathrm{pH}$ of solution and reaction temperature. The linear relationships between $\ln ([P S]), \ln ([O G]), \ln ([N-P C])$ and $\ln \left(k_{a p p}\right)$ were established. As shown in Fig. 6a-b, when the dose of PANI and PPy increase from 0.10 to 0.40 $\mathrm{g} / \mathrm{L}$, the degradation efficiency of $O G$ is significantly enhanced, as justified by the linear increase in $K_{a p p}$. This positive effect was due to the generation of more active sites in PANI or PPy used to activate PS, thus, producing more reactive species including $\mathrm{SO}_{4}{ }^{\circ-}$ and $\mathrm{OH}^{\circ}$, which significantly increase the OG degradation efficiency. In the same way, when, the concentration of the PS increases from 2 to $8 \mathrm{mM}$, the OG degradation rate increases linearly. In fact, as the persulfate amount increases, the degradation reaction becomes faster, since PPy and PANI 
catalysts have sufficient interactions with $\mathrm{S}_{2} \mathrm{O}_{8}{ }^{2}$, which favour the generation of radical species to degrade OG. However, a negative correlation between the $K_{a p p}$ and the $O G$ concentration was obtained as shown in Fig. $\mathbf{6 a - b}$. This fact could be related with the low amount of radicals available with regard to the high pollutant concentration. As reported from previous studies, the initial pH of solution can greatly affect the processes of PS activation [7]. Therefore, the development of metal free catalysts for high PS activation over a wide $\mathrm{pH}$ range is of great importance. As shown in Fig. 6c, the performance of both PANI/PS and PPy/PS systems is not $\mathrm{pH}$ dependent and suggested that the process can function within a wide $\mathrm{pH}$ range from 3 to 9. In fact, the acidic $\mathrm{pH}$ facilitates the protonation of nitrogen species present on the polymer catalyst surface resulting hence in a positive charge on the surface of the material which will facilitate the interaction occurring between the positively charged catalysts and the negatively charged PS $\left(\mathrm{S}_{2} \mathrm{O}_{8}{ }^{2-}\right)[7]$. The $\mathrm{pH}$ pzc value was determined by the projection of the $\Delta \mathrm{pH}=\mathrm{pH}_{\mathrm{f}}-$ $\mathrm{pH}_{\mathrm{i}}$ on $\mathrm{pH}_{\mathrm{i}}$, as a result, the $\mathrm{PZC}$ is the $\mathrm{pH}$ at which $\triangle \mathrm{pH}$ is zero. As shown in Fig. $6 \mathrm{~d}$, the PANI and PPy catalysts show pHpzc of 5.75 and 6.12, respectively. The obtained results are in agreement with the previous reported data $[39,40]$. Both PANI and PPy surfaces are positively charged when the $\mathrm{pH}$ solution was equal to 5.21 , due to the chemical amine groups protonation, promoting hence high electrostatic attractions between the negative charges of OG, as well as the PS and the positively charged PANi and PPy surfaces. In addition, the decrease in degradation rate at $\mathrm{pH} 3.15$ is due to the stabilization of $\mathrm{H}^{+}$ions with $\mathrm{SO}_{4}{ }^{2-}$ or $\mathrm{S}_{2} \mathrm{O}_{8}{ }^{2-}$ to form $\mathrm{HS}_{2} \mathrm{O}_{8}{ }^{-}$or $\mathrm{HSO}_{4}{ }^{-}$, which limit the generation of $\mathrm{SO}_{4}{ }^{--}$radicals. In alkaline medium, excess amount of $\mathrm{OH}^{-}$ions compete with the negatively charged anionic species (OG, $\mathrm{SO}_{4}{ }^{2-}$ and $\left.\mathrm{S}_{2} \mathrm{O}_{8}{ }^{2-}\right)$ resulting in the decreased degradation rate of $O G$. The effect of reaction temperature was also studied for the catalytic reactions PPy/PS and PANI/PS, to determine the activation energy of $O G$ degradation. Since the rates of the catalytic reactions were found to have positive effects on the OG degradation at elevated temperatures (Fig. S5a,b). The reaction temperatures selected for the experimental tests are $25^{\circ} \mathrm{C}, 35^{\circ} \mathrm{C}$ and $45{ }^{\circ} \mathrm{C}$. The relationship between the rate constant and the reaction temperature are shown in Fig. S5. The activation energy $\left(E_{a}\right)$ calculated from Arrhenius equation for the OG degradation was found to be equal to $28.7 \mathrm{~kJ} \mathrm{~mol}^{-1}$ for the PANI/PS system and $24.15 \mathrm{~kJ} \mathrm{~mol}^{-1}$ for the PPy/PS system. Moreover, the Ea value calculated in this study was lower than those reported previously (Table S3). 

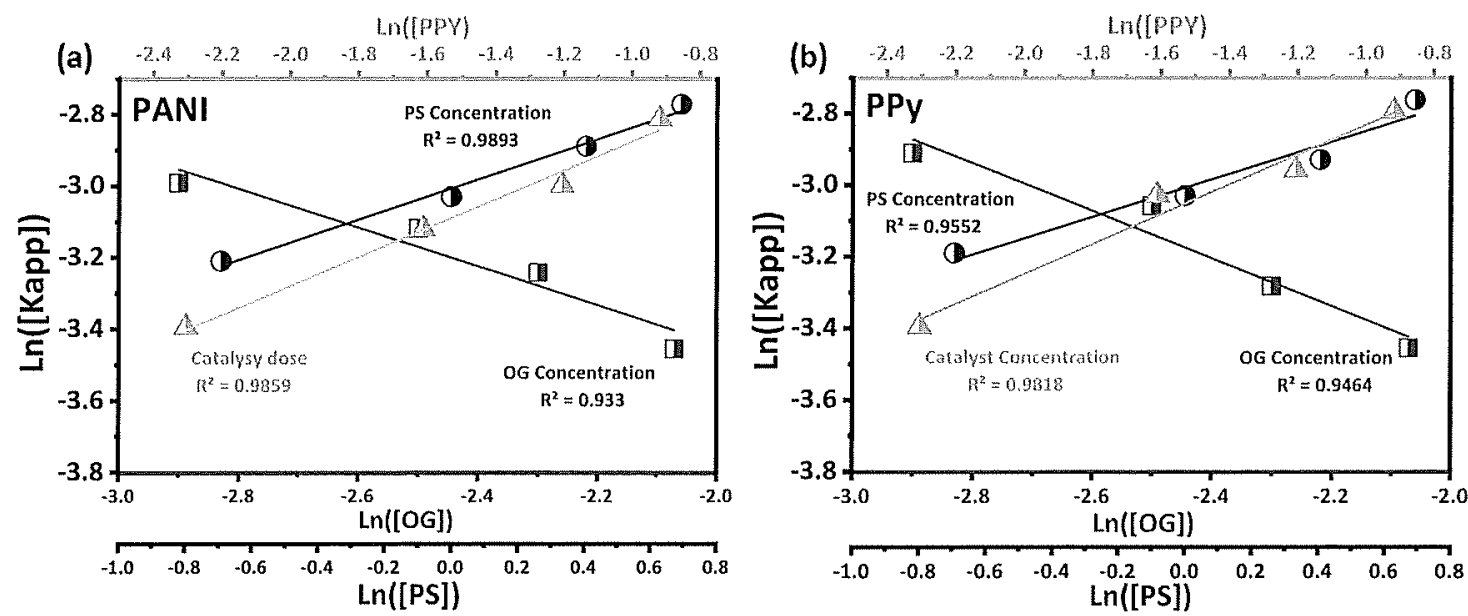

(c)
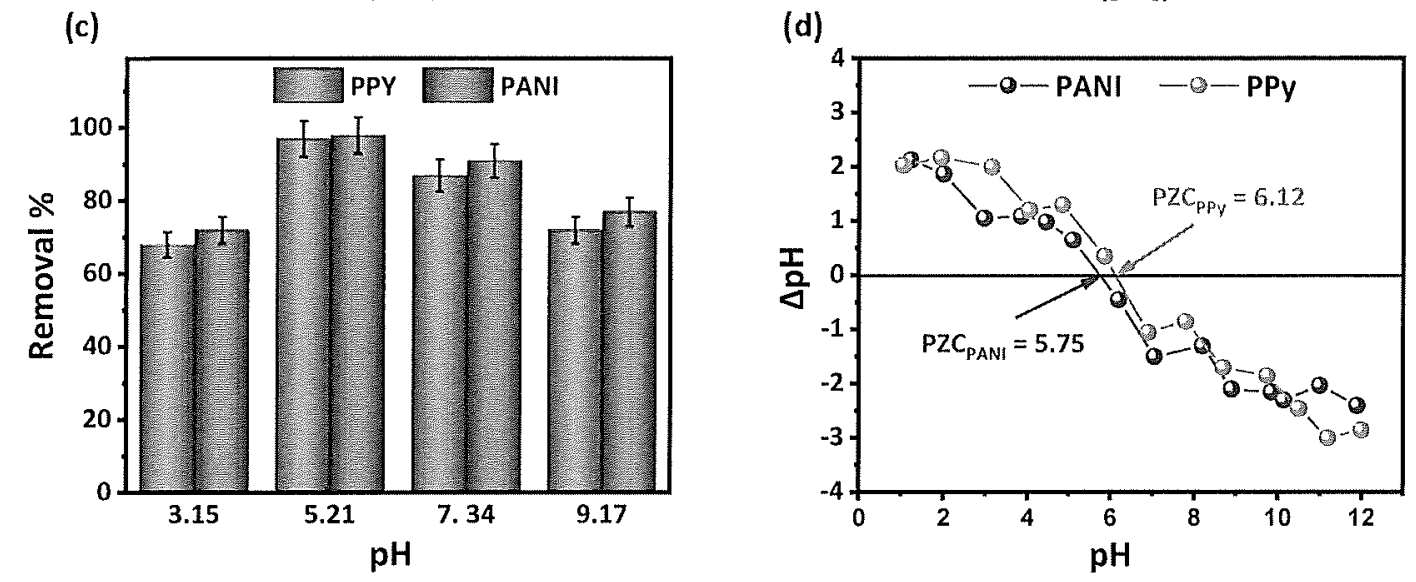

Fig. 6. Linear correlations between catalyst dose, PS concentration, OG concentration and $k_{\text {app }}$ in: PANI/PS (a) and PPY/PS (b) process; Effect of initial pH solution (c) ([catalyst] $=0.1$, $0.2,0.3,0.4 \mathrm{~g} \mathrm{~L}^{-1},[\mathrm{OG}]=50,75,100,125 \mathrm{mg} \mathrm{L}^{-1},[\mathrm{PS}]=, 3,4,5,6 \mathrm{mM}$ ); Point zero charge of PANI and PPy (d)

\subsection{Reactive Oxygen Species (ROS) Analysis and Activation Mechanisms}

Radical scavengers experiments were performed in order to identify the main reactive species in the reaction system. The different scavenging reagents used are ethanol (EtOH), tert-butyl alcohol (TBA), sodium azide ( $\left.\mathrm{NaN}_{3}\right)$. EtOH ( $\alpha$-hydrogen) is highly reactive towards $\mathrm{SO}_{4}{ }^{\circ}$ and $\mathrm{OH}^{\bullet}$ radicals. The reaction rate of $\left(\mathrm{OH}^{\bullet}\right)\left(\mathrm{k}=(1.2-2.8) \times 10^{9} \mathrm{M}^{-1} \mathrm{~s}^{-1}\right)$ and $\left(\mathrm{SO}_{4}{ }^{\circ}\right)(\mathrm{k}=$ $\left.(1.6-7.7) \times 10^{7} \mathrm{M}^{-1} \mathrm{~s}^{-1}\right)[16,18]$. Regarding $t$-BA, it reacts with a reaction rate of up to 1000 times with $\mathrm{OH}^{\bullet}\left(\mathrm{k}=(3.8-7.6) \times 10^{8}\right)$ than $\mathrm{SO}_{4}{ }^{--}\left(\mathrm{k}=(4.0-9.1) \times 10^{5} \mathrm{M}^{-1} \mathrm{~s}^{-1}\right)$, so it was used primarily to trap $\mathrm{OH}^{*}$ in an efficient manner $[18,28]$. For non-radical species, $\mathrm{NaN}_{3}$ is used as an excellent quencher of ${ }^{1} \mathrm{O}_{2},[14,34,43]$. As shown in Fig. 7a,d, in all systems, the OG degradation rate of the catalytic system did not decrease significantly with the addition of TBA, indicating that $\mathrm{HO}^{*}$ did not contribute significantly to the removal of OG because of their relatively low contents in the reaction system. However, the addition of EtOH in reaction 
system decreased the degradation of OG from $100 \%$ to $76 \%, 71 \%, 65 \%$ and $67 \%$ for PPy, PANI, PPy@Alg and PANI@Alg, respectively. These results indicate that $\mathrm{SO}_{4}{ }^{\circ-}$ are the preponderant reactive species for the OG degradation. On the other hand, the addition of low concentration of $\mathrm{NaN}_{3}$ to the reaction system inhibited the degradation of $\mathrm{OG}$ significantly. This indicates that the non-radical pathway $\left({ }^{1} \mathrm{O}_{2}\right)$ was predominant in the reaction system. Our data are in a good agreement with the reported works in the literature showing that non-radical processes can occur during the activation of PS by carbonaceous materials doped with nitrogen atoms $[41,42,44]$. The obtained results confirm that $\mathrm{SO}_{4}{ }^{--}$and ${ }^{1} \mathrm{O}_{2}$ were the main reactive species in all systems, and the degradation of OG was achieved by both radical and not radical pathways. Electron Paramagnetic Resonance (EPR) was applied as a powerful tool to further probe the generation and revolution processes of the main reactive species. PBN was used as the spin trapping reagent to directly detect the production of $\mathrm{SO}_{4}{ }^{--}$and ${ }^{\circ} \mathrm{OH}$ and TEMP were used as spin trapping agents to trap the ${ }^{1} \mathrm{O}_{2}[16,18]$. As shown in Fig. 7e,f, the EPR results provided evidences of the presence of $\mathrm{PBN}_{-} \mathrm{SO}_{4}{ }^{--}$and $\mathrm{PBN}-\mathrm{OH}^{\bullet}$ adducts in characteristic signals and revealed that both radicals were generated. When TEMP was used as the spin trapping agent, an obvious typical three-peaks signal with equal intensity was observed, which assigned to TEMP- ${ }^{1} \mathrm{O}_{2}$. The results indicate that ${ }^{1} \mathrm{O}_{2}$ might be the dominant reactive species responsible for OG degradation.
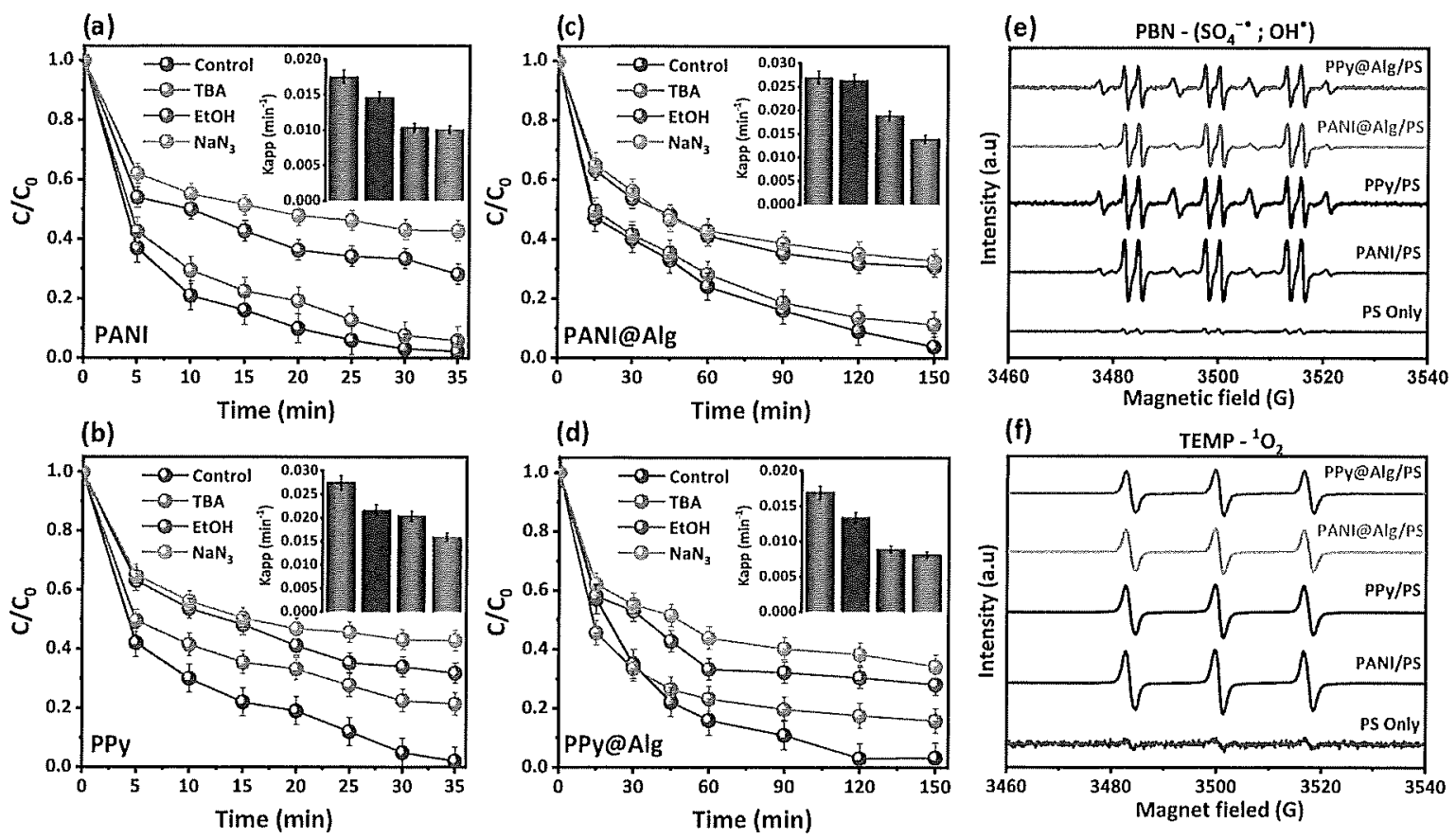
Fig. 7. Radical quenching results by using EtOH, TBA, and $\mathrm{NaN}_{3}$ as scavengers in (a)PANI/PS, (b)PPy/PS, (c)PANI@Alg/PS et (d)PPy@Alg/PS. (Experimental conditions: [Catalyst] = $0.2 \mathrm{~g} \mathrm{~L}^{-}$ $\left.1,[\mathrm{OG}]=50 \mathrm{mg} \mathrm{L}^{-1},[\mathrm{PS}]=4 \mathrm{mM},[\mathrm{EtOH}]=[\mathrm{TBA}]=1 \mathrm{M},\left[\mathrm{NaN}_{3}\right]=0.05 \mathrm{mM}\right) .(\mathrm{e}, \mathrm{f})$ EPR spectra using PBN and TEMP as the spin-trapping agents

The mechanism of the degradation process remains questionable and it should be elucidated in the future. However, when the carbon matrix catalyst is nitrogen doped, a nonradical mechanism can appear and plays the main role during the catalytic reaction $[14,47]$. The stability of the nitrogen atoms in the catalyst structure is a very important parameter for catalysts during the catalytic activation of PS. It should be noted that the loss of nitrogen sites is generally considered to be one of the reasons for the decrease in stability and deactivation of $\mathrm{N}$ doped carbon materials. Therefore, the stable $\mathrm{N}$ sites in both PPy and PANI polymers can provide good platforms in long-lasting catalytic reactions. The SEM images (Fig. S6a-b) of PANI and PPy after the catalytic reaction remain almost the same and comparable to fresh polymers, indicating that , the catalyst is not altered after the OG degradation reaction. Also, the FTIR analysis (Fig. S6c-d) demonstrates the stability of the PANI and PPy catalysts, as there is no change in the peaks of the two polymers after their uses. These results prove that the PANI and PPy catalysts are stable. Based on these results, the possible mechanism of activation of PS by the two conductive polymers PANI and PPy can be proposed as shown in Fig. 8a. Regarding the activation of PS by PANI (Fig. 8a), it was expressed by radical and nonradical routes, as confirmed by the obtained results of radical scavenging experiments and EPR analysis. Since the nitrogen electronegativity is greater than that of carbon $(\chi N=3.04 \mathrm{Vs}$ $\chi C=2.55$ ), the $\mathrm{S}_{2} \mathrm{O}_{8}{ }^{2-}$ will be adsorbed on the Nsites, by interaction occurring between the positively charged carbon domains and $\mathrm{S}_{2} \mathrm{O}_{8}{ }^{2-}$, and a reactive complex will be produced by intramolecular electron transfer, facilitating hence the $\mathrm{O}-\mathrm{O}$ bond cleavage. Further, the hydrogen atom transfer occurring in the polymer backbone, leads the $\mathrm{S}_{2} \mathrm{O}_{8}{ }^{2-}$ to liberate the $\mathrm{SO}_{4}{ }^{--}$radical, and converts the $-\mathrm{NH}_{2}{ }^{+}-/-\mathrm{NH}-$ bond to $=\mathrm{NH}^{+}-/=\mathrm{N}-$ (Eq. 1). Although the reaction occurring between $\mathrm{H}_{2} \mathrm{O}$ molecules and the $\mathrm{SO}_{4}{ }^{*}$ species could be responsible for the production of $\mathrm{OH}^{*}$ (Eq.3). In addition, the conjugated PANI system can promote the electrons transfer to sites such as $=\mathrm{NH}^{+}-/=\mathrm{N}-$ with higher transient oxidation state, and these sites, in turn, can react with $\mathrm{S}_{2} \mathrm{O}_{8}{ }^{2-}$ to produce reactive radicals. Note that one of the most reactive species in the catalytic reaction, ${ }^{1} \mathrm{O}_{2}$ can come from a non-radical species in the decomposition of PS (Eq. 1,3,4,6,7 and 8). Similarly, the activation of PS by PPy has been expressed by radical 
and non-radical pathways (Fig. 8a). In fact, PPy having a very high conductivity and characterized by rapid charge mobility, as well as strong affinity towards the PS, which promotes the electrons transfer. This polymer is also characterized by pyrrolic $\mathrm{N}(-\mathrm{NH}-)$ group which attracts the electrophilic species of PS and facilitates electron transfer. Moreover, the pyrrolic $\mathrm{N}(-\mathrm{NH}-)$ group of the PPy has an electron pair, and higher electronegativity, than that of carbon, thus allowing cleavage of the $\mathrm{O}-\mathrm{O}$ bond of $\mathrm{S}_{2} \mathrm{O}_{8}{ }^{2-}$ and producing reactive species. Note that the PPy conjugate system can cause electron transfer to $=\mathrm{N}^{\delta+} /=\mathrm{N}$ - with a higher transient oxidation state, then these sites can react with $\mathrm{S}_{2} \mathrm{O}_{8}{ }^{2-}$ to produce $\mathrm{SO}_{4}{ }^{-}$and $\mathrm{OH}^{*}$ (Eq. $2,3,5,6,7$, and 8 ). The adsorption of the PS on the pyrrolic $\mathrm{N}$ will lead to electrons exchange occurring between the adsorbed PS and the catalyst active sites, thus inducing a non-radical pathway.

Based on the catalytic activity results, the possible mechanism of PS activation by PANI/PPy@Alg alginate beads can be proposed as depicted in Fig. 8b. As shown below, the persulfate activation efficiency by both polymers will be depleted after surface coatings with alginate cross-linked with calcium ions; this will limit the diffusion of PS to the polymer active sites. The surface coating of polymers by alginate cross-linked with calcium ions will then reduce the electrons transfer due to lacking between the $\mathrm{N}$ conjugate atoms and $\mathrm{S}_{2} \mathrm{O}_{8}{ }^{2-}$ for PANI@Alg and the pyrrolic N/PS for PPy@Alg. Finally, all these radicals and non-radicals released will attack the organic pollutant molecules and will lead to a series of very complicated processes, such as rearrangement, cleavage of the $\mathrm{C}-\mathrm{C}$ bond, dehydrogenation for decomposition of organic molecules into $\mathrm{CO}_{2}$ and $\mathrm{H}_{2} \mathrm{O}$.

\section{Radical pathway}

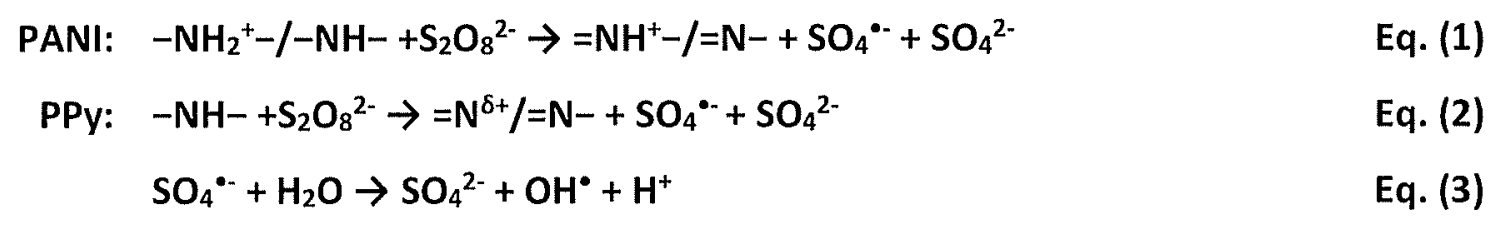

Non-Radical pathway

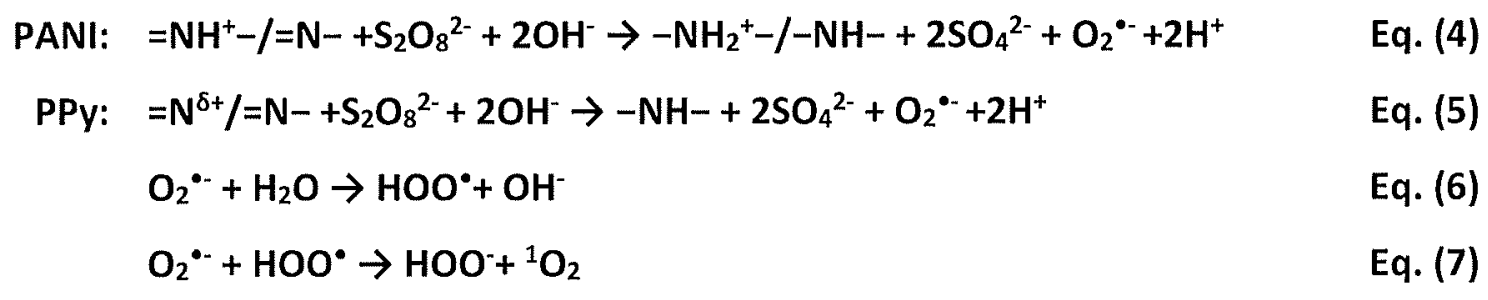




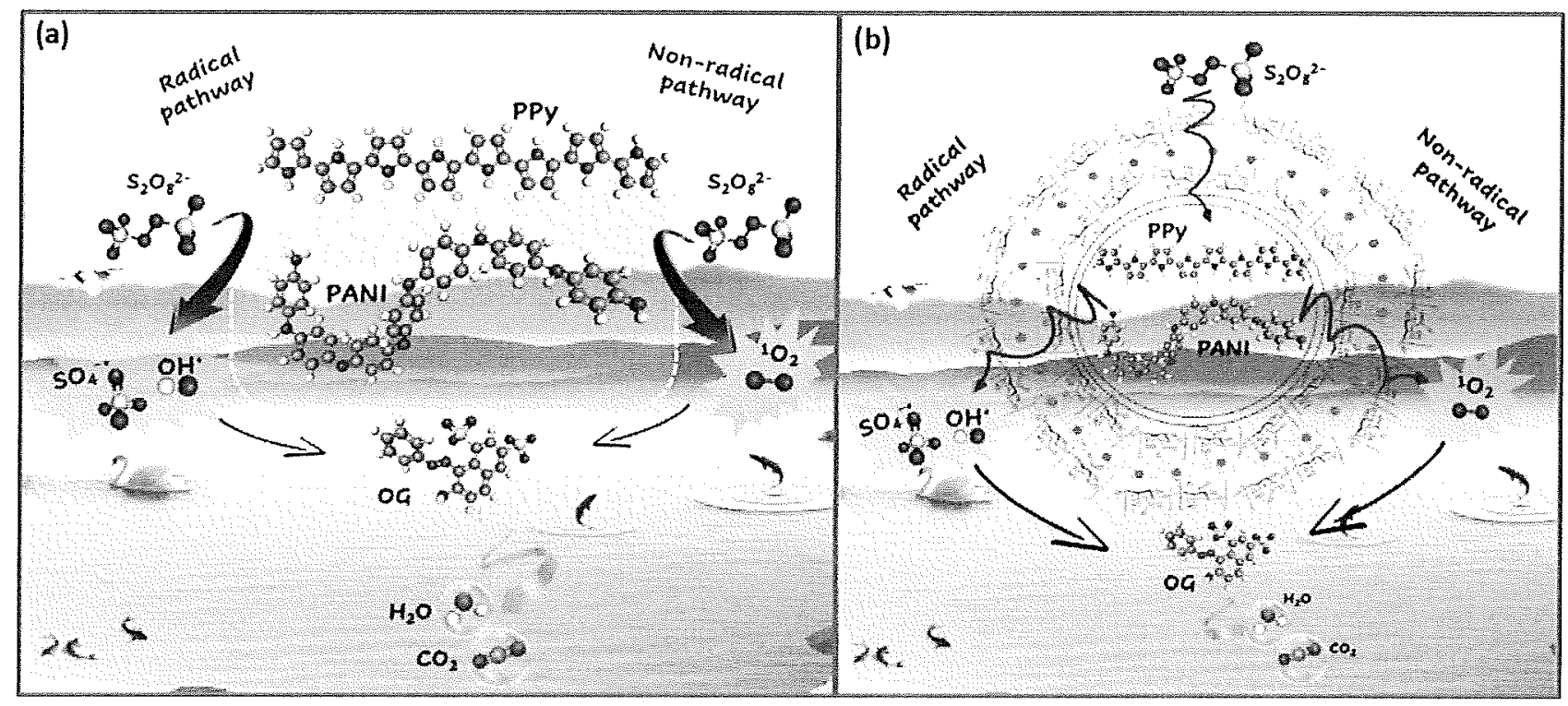

Fig. 8. Mechanism of the PS activation, on the PANI and PPy polymers (a); on the PANI@Alg and the PPy@Alg beads (b)

\subsection{Separation, Stability and Reusability}

The separation after use and the stability of the catalysts are the crucial factors in the practical environmental applications. These two problems may occur in the use of metal-free materials in the activation of persulfate for the degradation of organic pollutants. Therefore, to solve these problems we have prepared catalysts in the form of alginate beads to facilitate their separation after each cycle of use. In addition, we have followed the efficiency of the organic pollutant elimination after each of the five cycles used. Additionally, we have measured the mass losses for various catalysts: PANI and PPy alone, and PANI@Alg and PPy@Alg beads. As shown in Fig. 9a-b, after five cycles of reuse, the catalytic efficiency decreased slightly to 67 and 62\% for PANI and PPy respectively, whereas no decreases in the catalytic efficiency were observed by using PANI@Alg and PPy@Alg catalysts. The catalytic efficiency decreases of PANI and PPy polymers, after five cycles of reuse, may be attributed to the catalysts surface and structural changes, including loss of $\mathrm{N}$ atoms, adsorption of 
intermediates during degradation of organic molecules and coverage of active sites on the catalyst surface. However, PANI@Alg and PPy@Alg beads have efficient reusability during the five cycles, as shown in Fig. 9a-b. These results show that the catalysts in the form of the beads are more stable than the polymers alone, as resulting from the large structure of the beads, the ease of beads recovery from solutions and the decrease in the loss of $\mathrm{N}$ atoms after coating PANI and PPy with the complex of alginate and calcium ions. The new prepared hydrogel beads catalysts are stable, efficient, eco-friendly, easy to prepare, recyclable and thus have potential for industrial applications. Furthermore, after five cycles of liquid (OG) / solid (catalysts) filtration, the mass recovered were found to be: $0.135 \pm 0.001 \mathrm{~g} / \mathrm{L}, 0.140 \pm 0.001 \mathrm{~g} / \mathrm{L}, 0.240 \pm$ $0.001 \mathrm{~g} / \mathrm{L}$ and $0.245 \pm 0.001 \mathrm{~g} / \mathrm{L}$, for PANI,PPy, PANI@Alg and PPy@Alg, respectively. These results indicate that the alginate maintains very significantly the catalyst recovery, in comparison to the of the PANI and PPy alone (Fig.S7). Practically total recoveries were obtained in the case of PANI@Alg and PPy@Alg catalysts.
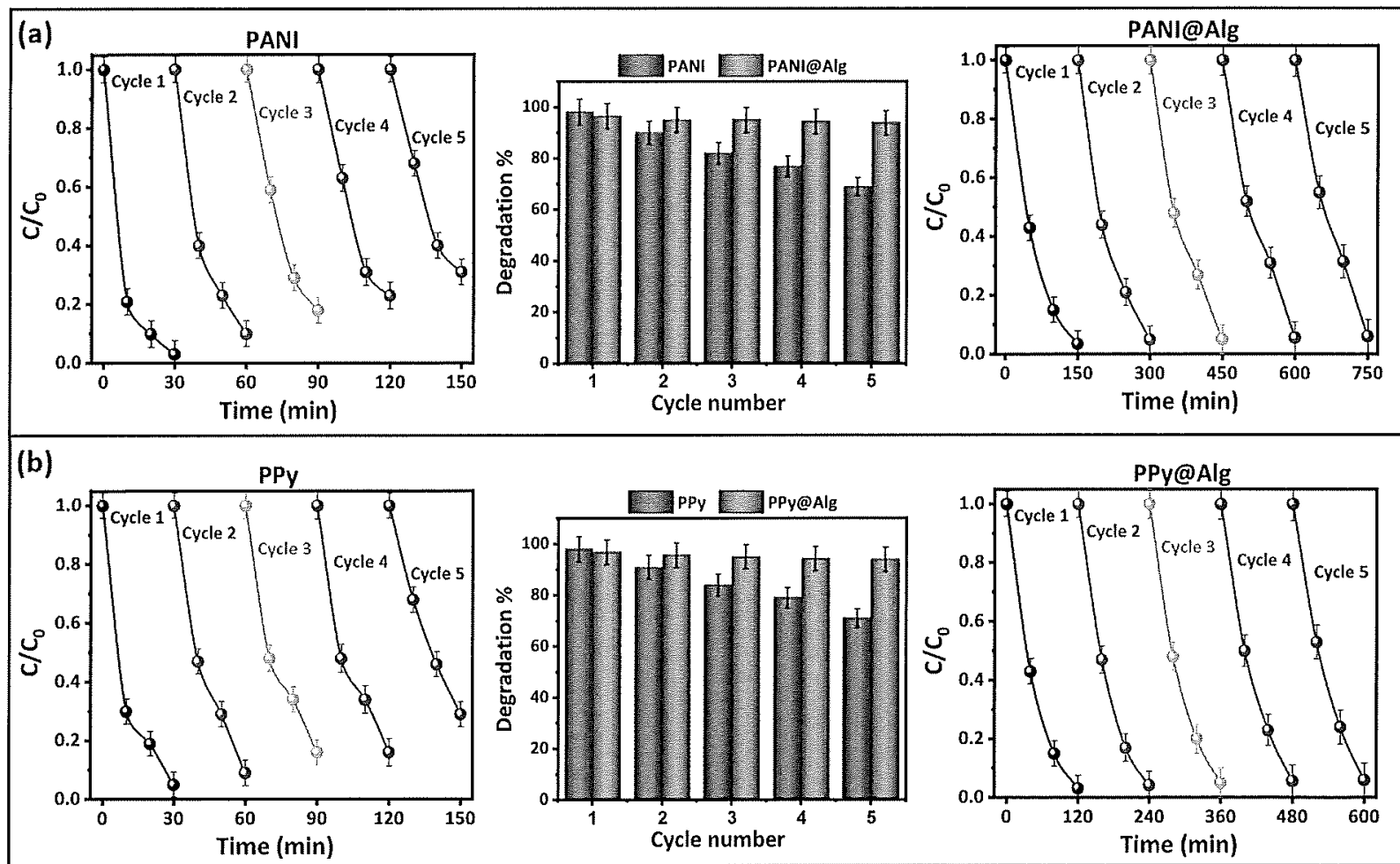

Fig.9. Stability and reusability of PANI and PANI@Alg (a), PPY and PPy@Alg (b); ([Catalyst] =

$$
\left.0.2 \mathrm{~g} \mathrm{~L}^{-1},[\mathrm{OG}]=50 \mathrm{mg} \mathrm{L}^{-1},[\mathrm{PS}]=4 \mathrm{mM}\right)
$$




\subsection{DFT Calculations}

In order to find a correlation between the electronic distribution of the studied monomers and their catalytic efficiency by using PS as an activator, parameters in terms of chemical descriptors were calculated based on the geometrical optimization of studied molecules (e.g. the energy of the highest occupied molecular orbital ( $E_{\text {Hомо }}$ ), the energy of the low unoccupied molecular orbital (ELUmo), energy gap $(\Delta E)$, hardness $(\eta)$, electronegativity $(\chi)$, and electrophilicity $(\omega))$. The values of the obtained parameters are shown in Table 1 and the frontier molecule orbital density are presented in Fig.S8.

Table 1. Electronic parameters of studied monomers PANI and PPY obtained by DFT/B3LYP 6-31g(d) calculations

\begin{tabular}{cccccccc}
\hline Catalyst & $\begin{array}{c}\mathrm{E}_{\text {Hомо }} \\
(\mathrm{eV})\end{array}$ & $\begin{array}{c}\mathrm{E}_{\text {LUMO }} \\
(\mathrm{eV})\end{array}$ & $\begin{array}{c}\Delta \mathrm{E} \\
(\mathrm{eV})\end{array}$ & $\begin{array}{c}\eta \\
(\mathrm{eV})\end{array}$ & $\begin{array}{c}\text { l } \\
(\mathrm{eV})\end{array}$ & $\begin{array}{c}\omega \\
(\mathrm{eV})\end{array}$ & $\begin{array}{c}\mathrm{E}_{\text {int }}(\mathrm{eV}) \\
\text { with } \\
\mathrm{S}_{2} \mathrm{O}_{8}{ }^{2-}\end{array}$ \\
\hline PANI & -4.9408 & -2.69257 & 2.248232 & 1.124116 & 3.816681 & 0.357784 & -1.68342 \\
\hline PPY & -4.88712 & -0.0366 & 4.850521 & 2.425261 & 2.461859 & 0.067359 & -1.07941 \\
\hline
\end{tabular}

In this part of the paper, various calculated parameters were assessed to elucidate the PS interaction with both studied molecules models (monomers of PANI and PPy). In fact, the high values of Eномо indicate the ability of the molecules to donate electrons. On the other hand, the lowest unoccupied molecular orbital energy values, Elumo indicate the capacity of the molecule to accept the electron $[48,49]$. From Table 1 we can clearly see that the PANI and the PPy show negative ELumo values, indicating, the charge transfer from PS to the polymer surface which proceeded easily from PPy $(-0.0366 \mathrm{eV})$ to PANI $(-2.69257 \mathrm{eV})$ during adsorption processes, according to the Frontier Molecule Orbital (FMO) theory of chemical reactivity [50]. Besides, the gap energy $\Delta \mathrm{E}=\mathrm{E}_{\text {LUMO }}-\mathrm{E}_{\text {HOM }}$ is relayed to the polarizability and the reactivity of monomers. This parameter $\Delta \mathrm{E}$ is found here to decrease in the order, PPy $>$ PANI, reflecting 
more affinity of the negative charge from the PS towards the PANI as compared to the PPy the polymer's surfaces.

The higher activation energy of PANI compared with PPy can be also explained by using The Hard-Soft-Acid-Base theory (HSAB). Generally, the less negative HOMO energy and the smaller energy gap for a given molecule result in stronger chemisorption binding [51]. The HSAB classifies reactive species as relatively "hard" or "Soft", based on polarizability, that is the ease with which electronic density can be displaced or delocalized to form new covalent bonds [52]. The softer molecules, with lowest $\eta$ value, are more reactive than the hard molecules having high $\eta$ value, because they require a higher energy for molecular excitation [53]. From Table 1, it was observed that the chemical hardness $(\eta)$ decreases from PPY to PANI which explains the highest activation energy of PANI. Other descriptors such as electrophilicity $(\omega)$ and electronegativity $(\chi)$ represent the electron pulling power of molecules and allow us to predict the reactivity of species to give or accept an electron. Therefore, the molecules with the lowest electronegativity values having the ability to easily give electrons and vice versa to molecules with high electronegativity values [54]. The obtained electronegativity and electrophilicity values of both monomers were presented in Table 1. However, the descriptors $(\omega)$ and $(\chi)$ present high values for both catalysts, with a high value for the PANI as compared to the PPY, which explains the ability to accept the PS negative charge in their surfaces.

The interaction energies (noted $E_{\text {int }}$ ) calculated according to D.C. Young [55] by using Eq (9) was also investigated. According to Table 1, the interaction energy calculated for both complexes PANI- $\mathrm{S}_{2} \mathrm{O}_{8}{ }^{2-}$ and $\mathrm{PPY}-\mathrm{S}_{2} \mathrm{O}_{8}{ }^{2-}$ show negative values, thus indicating the spontaneous adsorption process of PS on their surfaces (Fig. 10). In addition, the slight difference between the two interaction energies of the two complexes indicates an additional affinity of PS on the PANI surface as compared to PPY.

$$
E_{\text {int }}=E_{\text {catalyst } / P S}-E_{\text {catalyst }}-E_{P S} E_{q}(9)
$$

Where, $\mathbf{E}_{\mathbf{P S}}$ is the total energy of the PS atom, $\mathbf{E}_{\text {catalyst }}$ is the total energy of the PANI or PPY

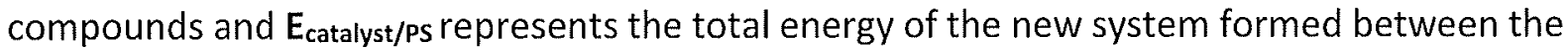
compounds studied and the PS. 


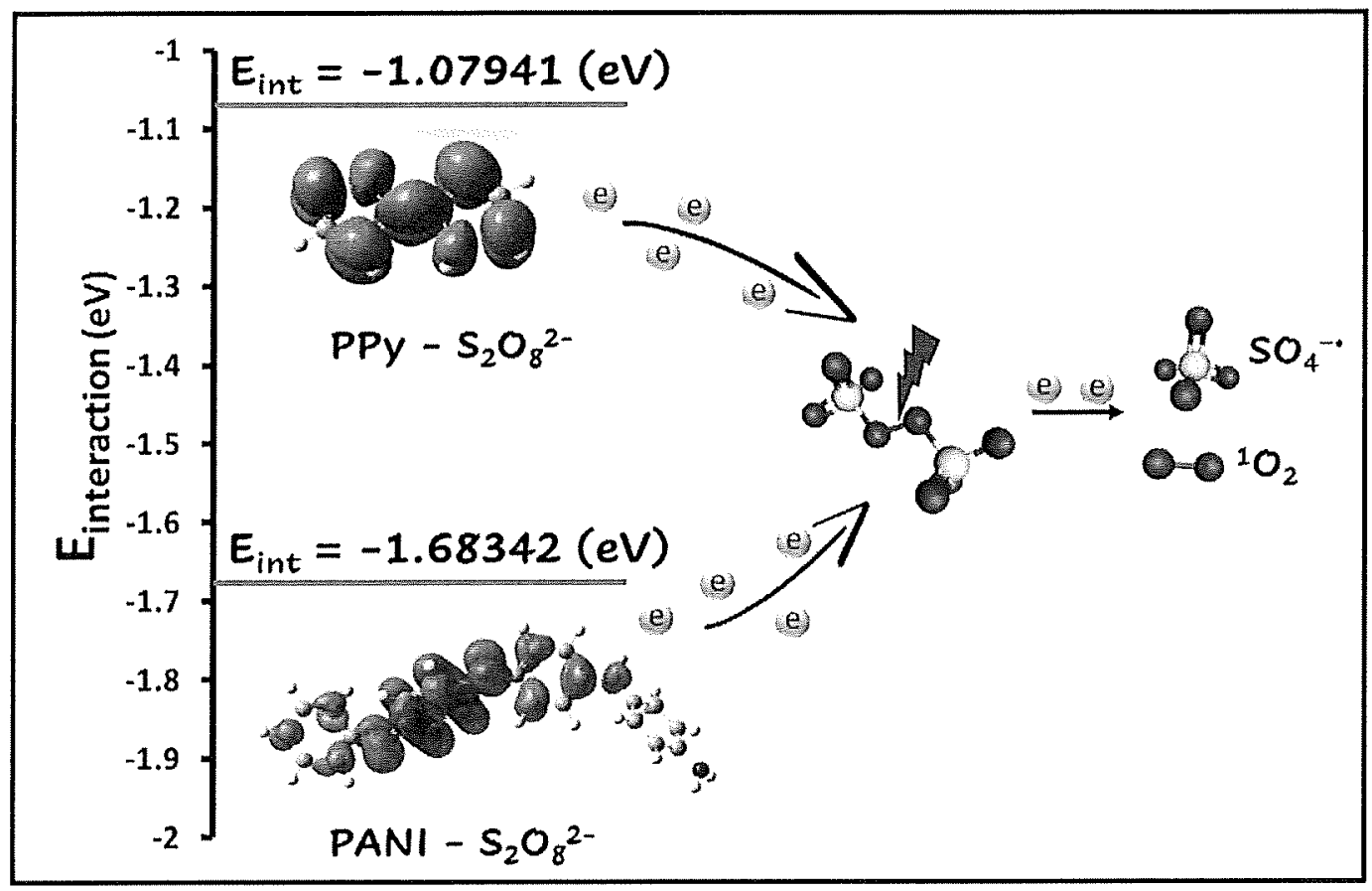

Fig. 10. Interaction Energies between the catalysts and the PS

\section{Conclusion}

In summary, conductive polymers such as Polyaniline (PANI) and Polypyrrole (PPy) were used as novel heterogeneous metal-free catalysts for their uses in the wastewater treatment. These polymers offer an effective new strategy to develop traditional metal-free catalysts for ecological sanitation and have excellent characteristics such as their stable $\mathrm{N}$ sites. The EPR spectra and the DFT calculations have further consolidated the role of $\mathrm{N}$ groups in the catalytic PS activation. Further, their conjugated chains prevent their structure oxidation, likely to occur during both the radical and no-radical catalytic reactions, generating the main reactive species such as $\mathrm{SO}_{4}{ }^{-{ }^{-}}$and ${ }^{1} \mathrm{O}_{2}$ toward degradation of organic pollutants. Therefore, in the present work we have developed stable and separable metal-free catalysts based on PANI and PPy polymers. The overall data have shown that PANI@Alg and PPy@Alg core-shell beads have excellent catalytic property for organic pollutants degradation. The approach used in this work provides an innovative method for the new prepared hydrogel beads catalysts, which are stable, efficient, eco-friendly, easy to prepare, recyclable and thus have potential for industrial applications. 


\section{CRediT author statement}

Abdellah AIT EL FAKIR (techniques of characterization) (Conceptualization) (Methodology) (Data curation) (writing - original draft), (interpretation). Zakaria ANFAR (Methodology) (Data curation) (review and editing) (techniques of characterization). Abdallah AMEDLous (Conceptualization), (interpretation), (review and editing). Mohamed ZBAIR (review and editing). Zakaria HAFIDI (Computational study), (review and editing), Mohammed EL ACHOURI (Computational study), (review and editing), Amane JADA (Project administration) (techniques of characterization) (Supervision) (Review and editing). Noureddine EL ALEM (Project administration) (Supervision) (review and editing).

\section{Acknowledgments}

Acknowledgment this work was supported by Franco-Moroccan cooperation framework, research project APUR 2019 between Laboratory of Materials \& Environment (LME), Ibn Zohr University, Agadir, Morocco, and Institute of Materials Science of Mulhouse (IS2M), Haute Alsace University, (IS2M-CNRS-UHA), Mulhouse, France. We thank, VAULOT Cyril, VIDAL Loïc, KNOPF Stéphan, FIOUX Philippe and MORLET - SAVARY Fabrice from IS2MMulhouse-France for the analyses of samples, respectively.

\section{References}

[1] Z. Anfar, R. El Haouti, S. Lhanafi, M. Benafqir, Y. Azougarh, N. El Alem, Treated digested residue during anaerobic co-digestion of Agri-food organic waste: Methylene blue adsorption, mechanism and CCD-RSM design, J. Environ. Chem. Eng. 5 (2017) 58575867. doi:10.1016/j.jece.2017.11.015.

[2] Z. Anfar, M. Zbair, H.A. Ahsaine, Y. Abdellaoui, A.A. El Fakir, E.H. Amaterz, A. Jada, N. El Alem, Preparation and Characterization of Porous Carbon@ZnO-NPs for Organic Compounds Removal: Classical Adsorption Versus Ultrasound Assisted Adsorption, ChemistrySelect. 4 (2019) 4981-4994. doi:10.1002/slct.201901043.

[3] Z. Anfar, A. Amedlous, A. Ait El Fakir, H. Ait Ahsaine, M. Zbair, S. Lhanafi, R. El Haouti, A. Jada, N. El Alem, Combined Methane Energy Recovery and Toxic Dye Removal by Porous Carbon Derived from Anaerobically Modified Digestate, ACS Omega. 4 (2019) 
9434-9445. doi:10.1021/acsomega.9b00524.

[4] E. Amaterz, A. Tara, A. Bouddouch, A. Taoufyq, B. Bakiz, A. Benlhachemi, O. Jbara, Photo-electrochemical degradation of wastewaters containing organics catalysed by phosphate-based materials: a review, Rev. Environ. Sci. Bio/Technology. 19 (2020) 843872. doi:10.1007/s11157-020-09547-9.

[5] L. Zhu, J. Ji, J. Liu, S. Mine, M. Matsuoka, J. Zhang, M. Xing, Designing 3D-MoS 2 Sponge as Excellent Cocatalysts in Advanced Oxidation Processes for Pollutant Control, Angew. Chemie. 132 (2020) 14072-14080. doi:10.1002/ange.202006059.

[6] D. Ding, S. Yang, X. Qian, L. Chen, T. Cai, Nitrogen-doping positively whilst sulfur-doping negatively affect the catalytic activity of biochar for the degradation of organic contaminant, Appl. Catal. B Environ. $263 \quad$ (2020) 118348. doi:10.1016/j.apcatb.2019.118348.

[7] J. Yu, H. Feng, L. Tang, Y. Pang, G. Zeng, Y. Lu, H. Dong, J. Wang, Y. Liu, C. Feng, J. Wang, B. Peng, S. Ye, Metal-free carbon materials for persulfate-based advanced oxidation process: Microstructure, property and tailoring, Prog. Mater. Sci. 111 (2020) 100654. doi:10.1016/j.pmatsci.2020.100654.

[8] J. Lee, U. von Gunten, J.-H. Kim, Persulfate-Based Advanced Oxidation: Critical Assessment of Opportunities and Roadblocks, Environ. Sci. Technol. 54 (2020) 30643081. doi:10.1021/acs.est.9b07082.

[9] P. Sun, H. Liu, M. Feng, L. Guo, Z. Zhai, Y. Fang, X. Zhang, V.K. Sharma, Nitrogen-sulfur co-doped industrial graphene as an efficient peroxymonosulfate activator: Singlet oxygen-dominated catalytic degradation of organic contaminants, Appl. Catal. B Environ. 251 (2019) 335-345. doi:10.1016/j.apcatb.2019.03.085.

[10] X. Chen, X. Duan, W.-D. Oh, P.-H. Zhang, C.-T. Guan, Y.-A. Zhu, T.-T. Lim, Insights into nitrogen and boron-co-doped graphene toward high-performance peroxymonosulfate activation: Maneuverable N-B bonding configurations and oxidation pathways, Appl. Catal. B Environ. 253 (2019) 419-432. doi:10.1016/j.apcatb.2019.04.018.

[11] X. Duan, Z. Ao, H. Zhang, M. Saunders, H. Sun, Z. Shao, S. Wang, Nanodiamonds in sp 2 /sp 3 configuration for radical to nonradical oxidation: Core-shell layer dependence, 
Appl. Catal. B Environ. 222 (2018) 176-181. doi:10.1016/j.apcatb.2017.10.007.

[12] J. Tan, Z. Li, J. Li, J. Wu, X. Yao, T. Zhang, Graphitic carbon nitride-based materials in activating persulfate for aqueous organic pollutants degradation: A review on materials design and mechanisms, Chemosphere. $262 \quad$ (2021) 127675. doi:10.1016/j.chemosphere.2020.127675.

[13] H. Wang, W. Guo, B. Liu, Q. Si, H. Luo, Q. Zhao, N. Ren, Sludge-derived biochar as efficient persulfate activators: Sulfurization-induced electronic structure modulation and disparate nonradical mechanisms, Appl. Catal. B Environ. 279 (2020) 119361. doi:10.1016/j.apcatb.2020.119361.

[14] J. Yu, L. Tang, Y. Pang, G. Zeng, H. Feng, J. Zou, J. Wang, C. Feng, X. Zhu, X. Ouyang, J. Tan, Hierarchical porous biochar from shrimp shell for persulfate activation: A twoelectron transfer path and key impact factors, Appl. Catal. B Environ. 260 (2020) 118160. doi:10.1016/j.apcatb.2019.118160.

[15] Q. Yang, Y. Chen, X. Duan, S. Zhou, Y. Niu, H. Sun, L. Zhi, S. Wang, Unzipping carbon nanotubes to nanoribbons for revealing the mechanism of nonradical oxidation by carbocatalysis, Appl. Catal. B Environ. $276 \quad$ (2020) 119146. doi:10.1016/j.apcatb.2020.119146.

[16] Z. Anfar, A.A. El Fakir, M. Zbair, Z. Hafidi, A. Amedlous, M. Majdoub, S. Farsad, A. Amjlef, A. Jada, N. El Alem, New functionalization approach synthesis of Sulfur doped, Nitrogen doped and Co-doped porous carbon: Superior metal-free Carbocatalyst for the catalytic oxidation of aqueous organics pollutants, Chem. Eng. J. 405 (2021) 126660. doi:10.1016/j.cej.2020.126660.

[17] E.-T. Yun, G.-H. Moon, H. Lee, T.H. Jeon, C. Lee, W. Choi, J. Lee, Oxidation of organic pollutants by peroxymonosulfate activated with low-temperature-modified nanodiamonds: Understanding the reaction kinetics and mechanism, Appl. Catal. B Environ. 237 (2018) 432-441. doi:10.1016/j.apcatb.2018.04.067.

[18] Z. Anfar, A. Ait El Fakir, H. Ait Ahsaine, M. Zbair, S. Farsad, F. Morlet-Savary, A. Jada, N. El Alem, Nitrogen doped graphitic porous carbon from almond shells as an efficient persulfate activator for organic compound degradation, New J. Chem. 44 (2020) 9391- 
9401. doi:10.1039/DONJ01148G.

[19] W. Ren, G. Nie, P. Zhou, H. Zhang, X. Duan, S. Wang, The Intrinsic Nature of Persulfate Activation and N-Doping in Carbocatalysis, Environ. Sci. Technol. 54 (2020) 6438-6447. doi:10.1021/acs.est.0c01161.

[20] M. Ghavami, M.Z. Kassaee, R. Mohammadi, M. Koohi, B.N. Haerizadeh, Polyaniline nanotubes coated with TiO2\&p-Fe2O3@graphene oxide as a novel and effective visible light photocatalyst for removal of rhodamine B from water, Solid State Sci. 38 (2014) 143-149. doi:10.1016/j.solidstatesciences.2014.09.010.

[21] V. Eskizeybek, F. Sari, H. Gülce, A. Gülce, A. Avci, Preparation of the new polyaniline/ZnO nanocomposite and its photocatalytic activity for degradation of methylene blue and malachite green dyes under UV and natural sun lights irradiations, Appl. Catal. B Environ. 119-120 (2012) 197-206. doi:10.1016/j.apcatb.2012.02.034.

[22] A. Jada, H. Debih, M. Khodja, Montmorillonite surface properties modifications by asphaltenes adsorption, J. Pet. Sci. Eng. 52 (2006) 305-316. doi:10.1016/j.petrol.2006.03.016.

[23] T. Mahmood, M.T. Saddique, A. Naeem, P. Westerhoff, S. Mustafa, A. Alum, Comparison of Different Methods for the Point of Zero Charge Determination of NiO, Ind. Eng. Chem. Res. 50 (2011) 10017-10023. doi:10.1021/ie200271d.

[24] K.L. Tan, B.T.G. Tan, E.T. Kang, K.G. Neoh, X-ray photoelectron spectroscopy studies of the chemical structure of polyaniline, Phys. Rev. B. 39 (1989) 8070-8073. doi:10.1103/PhysRevB.39.8070.

[25] C. Hennig, K.H. Hallmeier, R. Szargan, XANES investigation of chemical states of nitrogen in polyaniline, Synth. Met. 92 (1998) 161-166. doi:10.1016/S0379-6779(98)80106-9.

[26] J. Tabačiarová, M. Mičušík, P. Fedorko, M. Omastová, Study of polypyrrole aging by XPS, FTIR and conductivity measurements, Polym. Degrad. Stab. 120 (2015) 392-401. doi:10.1016/j.polymdegradstab.2015.07.021.

[27] J. Cao, Y. Wang, J. Chen, X. Li, F.C. Walsh, J.H. Ouyang, D. Jia, Y. Zhou, Three-dimensional graphene oxide/polypyrrole composite electrodes fabricated by one-step 
electrodeposition for high performance supercapacitors, J. Mater. Chem. A. 3 (2015) 14445-14457. doi:10.1039/c5ta02920a.

[28] B. Sun, W. Ma, N. Wang, P. Xu, L. Zhang, B. Wang, H. Zhao, K.-Y.A. Lin, Y. Du, Polyaniline: A New Metal-Free Catalyst for Peroxymonosulfate Activation with Highly Efficient and Durable Removal of Organic Pollutants, Environ. Sci. Technol. 53 (2019) 9771-9780. doi:10.1021/acs.est.9b03374.

[29] P.D. Bui, H.H. Tran, F. Kang, Y.-F. Wang, T.M. Cao, S.-J. You, N.H. Vu, V. Van Pham, Insight into the Photocatalytic Mechanism of Tin Dioxide/Polyaniline Nanocomposites for NO Degradation under Solar Light, ACS Appl. Nano Mater. 1 (2018) 5786-5794. doi:10.1021/acsanm.8b01445.

[30] M.T. Ramesan, Synthesis, characterization, and conductivity studies of polypyrrole/copper sulfide nanocomposites, J. Appl. Polym. Sci. (2012) n/a-n/a. doi:10.1002/app.38304.

[31] M. Alfatama, L.Y. Lim, T.W. Wong, Alginate-C18 Conjugate Nanoparticles Loaded in Tripolyphosphate-Cross-Linked Chitosan-Oleic Acid Conjugate-Coated Calcium Alginate Beads as Oral Insulin Carrier, Mol. Pharm. 15 (2018) 3369-3382. doi:10.1021/acs.molpharmaceut.8b00391.

[32] Y. Li, X. Zhao, Q. Xu, Q. Zhang, D. Chen, Facile Preparation and Enhanced Capacitance of the Polyaniline/Sodium Alginate Nanofiber Network for Supercapacitors, Langmuir. 27 (2011) 6458-6463. doi:10.1021/la2003063.

[33] K.M. Sajesh, R. Jayakumar, S. V. Nair, K.P. Chennazhi, Biocompatible conducting chitosan/polypyrrole-alginate composite scaffold for bone tissue engineering, Int. J. Biol. Macromol. 62 (2013) 465-471. doi:10.1016/j.ijbiomac.2013.09.028.

[34] L. Tang, Y. Liu, J. Wang, G. Zeng, Y. Deng, H. Dong, H. Feng, J. Wang, B. Peng, Enhanced activation process of persulfate by mesoporous carbon for degradation of aqueous organic pollutants: Electron transfer mechanism, Appl. Catal. B Environ. 231 (2018) 110. doi:10.1016/j.apcatb.2018.02.059.

[35] Q. Huang, J. Zhang, Z. He, P. Shi, X. Qin, W. Yao, Direct fabrication of lamellar selfsupporting Co304/N/C peroxymonosulfate activation catalysts for effective aniline 
degradation, Chem. Eng. J. 313 (2017) 1088-1098. doi:10.1016/j.cej.2016.11.002.

[36] Y. Zhu, M. Yue, V. Natarajan, L. Kong, L. Ma, Y. Zhang, Q. Zhao, J. Zhan, Efficient activation of persulfate by Fe304@b- cyclodextrin nanocomposite for removal of bisphenol A, RSC Adv. 8 (2018) 14879-14887. doi:10.1039/c8ra01696h.

[37] X. Yue, W. Guo, X. Li, H. Zhou, R. Wang, Core-shell Fe304@MIL-101(Fe) composites as heterogeneous catalysts of persulfate activation for the removal of Acid Orange 7, Environ. Sci. Pollut. Res. 23 (2016) 15218-15226. doi:10.1007/s11356-016-6702-5.

[38] Q. Ma, X. Zhang, R. Guo, H. Zhang, Q. Cheng, M. Xie, X. Cheng, Persulfate activation by magnetic $\psi$-Fe2O3/Mn304 nanocomposites for degradation of organic pollutants, Sep. Purif. Technol. 210 (2019) 335-342. doi:10.1016/j.seppur.2018.06.060.

[39] L. Hlekelele, N.E. Nomadolo, K.Z. Setshedi, L.E. Mofokeng, A. Chetty, V.P. Chauke, Synthesis and characterization of polyaniline, polypyrrole and zero-valent iron-based materials for the adsorptive and oxidative removal of bisphenol-A from aqueous solution, RSC Adv. 9 (2019) 14531-14543. doi:10.1039/C9RA01666J.

[40] R. Li, L. Liu, F. Yang, Preparation of polyaniline/reduced graphene oxide nanocomposite and its application in adsorption of aqueous Hg(II), Chem. Eng. J. 229 (2013) 460-468. doi:10.1016/j.cej.2013.05.089.

[41] X. Huo, P. Zhou, J. Zhang, Y. Liu, X. Cheng, Y. Liu, W. Li, Y. Zhang, N, S-Doped porous carbons for persulfate activation to remove tetracycline: Nonradical mechanism, J. Hazard. Mater. 391 (2020) 122055. doi:10.1016/j.jhazmat.2020.122055.

[42] S. Liu, C. Lai, B. Li, C. Zhang, M. Zhang, D. Huang, L. Qin, H. Yi, X. Liu, F. Huang, X. Zhou, L. Chen, Role of radical and non-radical pathway in activating persulfate for degradation of p-nitrophenol by sulfur-doped ordered mesoporous carbon, Chem. Eng. J. 384 (2020) 123304. doi:10.1016/j.cej.2019.123304.

[43] S. Ye, G. Zeng, X. Tan, H. Wu, J. Liang, B. Song, N. Tang, P. Zhang, Y. Yang, Q. Chen, X. Li, Nitrogen-doped biochar fiber with graphitization from Boehmeria nivea for promoted peroxymonosulfate activation and non-radical degradation pathways with enhancing electron transfer, Appl. Catal. B Environ. $269 \quad$ (2020) 118850. doi:10.1016/j.apcatb.2020.118850. 
[44] J. Miao, W. Geng, P.J.J. Alvarez, M. Long, 2D N-Doped Porous Carbon Derived from Polydopamine-Coated Graphitic Carbon Nitride for Efficient Nonradical Activation of Peroxymonosulfate, Environ. Sci. Technol. $54 \quad$ (2020) 8473-8481. doi:10.1021/acs.est.0c03207.

[45] S. Liu, C. Lai, B. Li, C. Zhang, M. Zhang, D. Huang, L. Qin, H. Yi, X. Liu, F. Huang, X. Zhou, L. Chen, Role of radical and non-radical pathway in activating persulfate for degradation of p-nitrophenol by sulfur-doped ordered mesoporous carbon, Chem. Eng. J. 384 (2020) 123304. doi:10.1016/j.cej.2019.123304.

[46] J. Yu, L. Tang, Y. Pang, G. Zeng, H. Feng, J. Zou, J. Wang, C. Feng, X. Zhu, X. Ouyang, J. Tan, Hierarchical porous biochar from shrimp shell for persulfate activation: A twoelectron transfer path and key impact factors, Appl. Catal. B Environ. 260 (2020) 118160. doi:10.1016/j.apcatb.2019.118160.

[47] Y. Shi, J. Zhu, G. Yuan, G. Liu, Q. Wang, W. Sun, B. Zhao, L. Wang, H. Zhang, Activation of persulfate by EDTA-2K-derived nitrogen-doped porous carbons for organic contaminant removal: Radical and non-radical pathways, Chem. Eng. J. 386 (2020) 124009. doi:10.1016/j.cej.2019.124009.

[48] G. Gece, The use of quantum chemical methods in corrosion inhibitor studies, Corros. Sci. 50 (2008) 2981-2992. doi:10.1016/j.corsci.2008.08.043.

[49] K. Fukui, Theory of Orientation and Stereoselection, Springer Berlin Heidelberg, Berlin, Heidelberg, 1975. doi:10.1007/978-3-642-61917-5.

[50] M.J.S. Dewar, A critique of frontier orbital theory, J. Mol. Struct. THEOCHEM. 200 (1989) 301-323. doi:10.1016/0166-1280(89)85062-6.

[51] F. Melnikov, B.C. Geohagen, T. Gavin, R.M. LoPachin, P.T. Anastas, P. Coish, D.W. Herr, Application of the hard and soft, acids and bases (HSAB) theory as a method to predict cumulative neurotoxicity, Neurotoxicology. $79 \quad$ (2020) 95-103. doi:10.1016/j.neuro.2020.04.009.

[52] T.-L. Ho, Hard soft acids bases (HSAB) principle and organic chemistry, Chem. Rev. 75 (1975) 1-20. doi:10.1021/cr60293a001. 
[53] R.P. Iczkowski, J.L. Margrave, Electronegativity, J. Am. Chem. Soc. 83 (1961) 3547-3551. doi:10.1021/ja01478a001.

[54] H.O. Pritchard, H.A. Skinner, The Concept Of Electronegativity, Chem. Rev. 55 (1955) 745-786. doi:10.1021/cr50004a005.

[55] D.C. Young, Computational Chemistry, John Wiley \& Sons, Inc., New York, USA, 2001. doi:10.1002/0471220655. 